\title{
Tratamientos para la Remoción de Metales Pesados Comúnmente Presentes en Aguas Residuales Industriales. Una Revisión
}

\section{Treatments for Removal of Heavy Metals Commonly Found in Industrial Wastewater. A Review}

Diego Ivan Caviedes Rubio ${ }^{[1]}$, Ramiro Adolfo Muñoz Calderón ${ }^{[2]}$, Alexandra Perdomo Gualtero ${ }^{[3]}$, Daniel Rodríguez Acosta ${ }^{[4]}$ y Ivan Javier Sandoval Rojas ${ }^{[5]}$

\begin{abstract}
Resumen
Este artículo presenta una revisión de algunas características toxicológicas de metales pesados, sus fuentes industriales, los niveles permisivos de vertimiento y 20 diferentes técnicas subdivididas en convencionales y no convencionales empleadas para la remoción de metales pesados en medios hídricos, así como las condiciones fisicoquímicas en las que estos tratamientos han presentado mejores eficiencias de remoción.
\end{abstract}

Palabras clave: Metales Pesados; Aguas Residuales; Condiciones experimentales; Técnicas convencionales y no convencionales.

\begin{abstract}
This article presents an overview of some toxicological characteristics of heavy metals, their industrial sources, permissive levels of discharge, and 20 different techniques subdivided into conventional and unconventional treatments used for the removal of heavy metals in water sources. The paper also analyzes the physicochemical conditions in which these treatments have presented higher removal efficiencies.
\end{abstract}

Key words: heavy metals; wastewater; experimental conditions; conventional and unconventional techniques.

[1] Magister en Ecología y Gestión de Ecosistemas Estratégicos. Docente Universidad Cooperativa de Colombia. Grupo de Investigación en Administración de Operaciones y Producción ADOPRO. E-mail: diego.caviedesr@campusucc. edu.co

[2] Ingeniero civil. Subgerente Técnico y Operativo Aguas del Huila S.A.-E.S.P. Neiva. Calle 21 No. 1C-17 ramiromunoz@ live.com

[3] Licenciada en Ciencias Naturales y Educación Ambiental. Tutora Corporación Infancia y Desarrollo. alexandra.1455@ hotmail.com

[4] Ingeniero Forestal. Instructor Servicio Nacional de Aprendizaje SENA. carrera7A con calle 6 esquina, danielsmithijo@ misena.edu.co

[5] Ingeniero Ambiental. Profesional Universitario CAM. Neiva. ivanjsandoval@gmail.com

Recibido: 16 mayo 2015 • Aceptado: 30 mayo 2015 


\section{Introducción}

Los elementos metálicos, debido a que están presentes en los ecosistemas acuáticos a muy baja concentración, son denominados elementos traza. Algunos son nutrientes esenciales para las plantas y los animales, micronutrientes (como Mn (Manganeso), Mo (Molibdeno), Cu (Cobre), Co (Cobalto), Zn (Zinc), Sc (Escandio) y V (Vanadio)) mientras que otros elementos (como el Ni (Níquel), Sn (Estaño) y Cr (Cromo)) son esenciales únicamente para los animales, pero cuando estos elementos están presentes en sistemas ambientales a concentraciones superiores a ciertos niveles, debido a desequilibrios naturales o por introducción antropogénica, pueden ser toxicos para los seres vivos (Domenech \& Peral, 2008). Otro término en la clasificación de los metales, es el de los metales pesados, refiriéndose a aquellos metales que causan un impacto ambiental debido a su toxicidad y cuya propiedad principal es tener una densidad aproximadamente superior a $6 \mathrm{~g} / \mathrm{cm}^{3}$ exceptuando al Ti (Titanio) (4,5 g/cc) pero también considerado como metal pesado y al As, que aunque es un no metal, presenta una alta densidad de $5,7 \mathrm{~g} / \mathrm{cm}^{3}$, por lo que se considera un metal pesado, además de otras propiedades de carácter ambiental (Baird, 2001; Domenech \& Peral, 2008; O’Connell, et al; 2008).
La implementación de metales en los procesos industriales han permitido que grandes concentraciones de metales pesados potencialmente tóxicos sean emitidos a la atmósfera y vertidos en los ambientes acuáticos y terrestres (Fu \& wang, 2011. O’Connell, et al; 2008); en el medio hidrosferico, cantidades cercanas a $10^{9} \mathrm{Kg}$ /año de metales traza se han vertido, siendo las aguas residuales de origen domésticas, las plantas térmicas, las fundiciones y las acerías, las principales fuentes de emisión(Tabla 1); el orden de flujos de emisión o cargas contaminantes de metales a la hidrosfera corresponde a $\mathrm{Mn}>\mathrm{Zn}>\mathrm{Cr}>$ $\mathrm{Pb}($ Plomo $)>\mathrm{Ni} \approx \mathrm{Cu}>\mathrm{Se}$ (Selenio) $\approx$ As (Arsénico) $>$ $\mathrm{Sb}$ (Antimonio) $>\mathrm{V} \approx \mathrm{Mo}$ ? Cd (Cadmio) $>\mathrm{Hg}$ (Mercurio) (Domenech \& Peral, 2008).

La toxicidad de los metales pesados depende de su movilidad en el medio, que a su vez, depende de su especiación química, persistencia y tendencia de acumulación o bioacumulacion (Domenech \& Peral, 2008. Kumar, et al; 2012). En la tabla 2 se describen los síntomas de la intoxicación típicos, la dosis letal en la dieta humana y los niveles de contaminación máximos (NCM) establecidos por la Agencia de Protección Ambiental de los Estados Unidos (USEPA) (Spiro, et al; 2006; Nguyen, et al; 2013.) y por la legislación Colombiana a través del Ministerio de Ambiente y Desarrollo Sostenible (MADS, 2015) para algunos metales pesados.

Tabla 1. Principales actividades industriales generadoras de metales pesados.

\begin{tabular}{l|l|l}
\hline \multicolumn{1}{c|}{ Industria } & \multicolumn{1}{c}{ Metales } & \multicolumn{1}{c}{ Contaminación derivada } \\
\hline $\begin{array}{l}\text { Minería de } \\
\text { metales ferrosos }\end{array}$ & $\mathrm{Cd}, \mathrm{Cu}, \mathrm{Ni}, \mathrm{Cr}, \mathrm{Co}, \mathrm{Zn}$ & Drenaje ácido de mina, relaves, escombreras \\
\hline $\begin{array}{l}\text { Extracción de } \\
\text { minerales }\end{array}$ & $\mathrm{As}, \mathrm{Cd}, \mathrm{Cu}, \mathrm{Ni}, \mathrm{Pb}, \mathrm{Zn}$ & Presencia en las menas como en los subproductos \\
\hline Fundición & $\mathrm{As}, \mathrm{Cd}, \mathrm{Pb}, \mathrm{Tl}$ & Procesado del mineral para obtención de metales \\
\hline Metalúrgica & $\mathrm{Cr}, \mathrm{Cu}, \mathrm{Mn}, \mathrm{Pb}, \mathrm{Sb}, \mathrm{Zn}$ & Procesado térmico de metales \\
\hline $\begin{array}{l}\text { Aleaciones y } \\
\text { aceros }\end{array}$ & $\mathrm{Pb}, \mathrm{Mo}, \mathrm{Ni}, \mathrm{Cu}, \mathrm{Cd}, \mathrm{As}, \mathrm{Te}, \mathrm{U}, \mathrm{Zn}$ & $\begin{array}{l}\text { Fabricación, eliminación y reciclaje de metales. } \\
\text { Relaves y escoriales }\end{array}$ \\
\hline $\begin{array}{l}\text { Gestión de } \\
\text { Residuos }\end{array}$ & $\mathrm{Zn}, \mathrm{Cu}, \mathrm{Cd}, \mathrm{Pb}, \mathrm{Ni}, \mathrm{Cr}, \mathrm{Hg}, \mathrm{Mn}$ & Incineración de residuos o en lixiviados \\
\hline $\begin{array}{l}\text { Corrosión } \\
\text { Metálica }\end{array}$ & $\mathrm{Fe}, \mathrm{Cr}, \mathrm{Pb}, \mathrm{Ni}, \mathrm{Co}, \mathrm{Zn}$ & $\begin{array}{l}\text { Inestabilidad de los metales expuestos al medio } \\
\text { ambiente }\end{array}$ \\
\hline Galvanoplastia & $\mathrm{Cr}, \mathrm{Ni}, \mathrm{Zn}, \mathrm{Cu}$ & Los efluentes líquidos de procesos de recubrimiento \\
\hline $\begin{array}{l}\text { Pinturas y } \\
\text { pigmentos }\end{array}$ & $\mathrm{Pb}, \mathrm{Cr}, \mathrm{As}, \mathrm{Ti}, \mathrm{Ba}, \mathrm{Zn}$ & $\begin{array}{l}\text { Residuos acuosos procedentes de la fabricación y el } \\
\text { deterioro de la pintura vieja. }\end{array}$ \\
\hline Baterías & $\mathrm{Pb}, \mathrm{Sb}, \mathrm{Zn}, \mathrm{Cd}, \mathrm{Ni}, \mathrm{Hg}$ & $\begin{array}{l}\text { Fluido de la pila de residuos, la contaminación del } \\
\text { suelo y las aguas subterráneas. }\end{array}$ \\
\hline Electrónica & $\mathrm{Pb}, \mathrm{Cd}, \mathrm{Hg}, \mathrm{Pt}, \mathrm{Au}, \mathrm{Cr}, \mathrm{As}, \mathrm{Ni}, \mathrm{Mn}$ & $\begin{array}{l}\text { Residuos metálicos acuosa y sólida desde el proceso de } \\
\text { fabricación y reciclaje }\end{array}$ \\
\hline $\begin{array}{l}\text { Agricultura y } \\
\text { Ganadería }\end{array}$ & $\begin{array}{l}\mathrm{Cd}, \mathrm{Cr}, \mathrm{Mo}, \mathrm{Pb}, \mathrm{U}, \mathrm{V}, \mathrm{Zn}, \mathrm{As}, \mathrm{Mn}, \\
\mathrm{Cu}\end{array}$ & $\begin{array}{l}\text { Contaminación de escorrentía, aguas superficiales y } \\
\text { subterráneas, la bioacumulacion planta }\end{array}$ \\
\hline
\end{tabular}


Tabla 2. Síntomas de la intoxicación típicos y los niveles de contaminación máximos (NCM) establecidos por (USEPA) y por la legislación Colombiana (Adaptado de la Secretaria Distrital de Ambiente Bogotá (SDAB), 2010; Nguyen, et al; 2013; MADS, 2015).

\begin{tabular}{|c|c|c|c|c|}
\hline \multirow{2}{*}{$\begin{array}{l}\text { Metal } \\
\text { Pesado }\end{array}$} & \multirow{2}{*}{ Toxicidad } & \multirow{2}{*}{$\begin{array}{l}\text { Dosis letal en dieta } \\
\text { humana } \mathrm{mg} / \text { día }\end{array}$} & \multicolumn{2}{|c|}{$\mathrm{NCM}(\mathrm{mg} / \mathrm{L})$} \\
\hline & & & USEPA & Colombia \\
\hline As & $\begin{array}{l}\text { Manifestaciones cutáneas, canceres viscerales, } \\
\text { enfermedad vascular }\end{array}$ & $50-340$ & 0,05 & $0,1-0,5$ \\
\hline Cd & Daño renal, trastorno renal y efectos carcinógenos & $1,5 \mathrm{k}-9 \mathrm{k}$ & 0,01 & 0,1 \\
\hline $\mathrm{Cr}$ & $\begin{array}{l}\text { Dolor de cabeza, diarrea, náuseas, vomito, } \\
\text { carcinógeno }\end{array}$ & $3 \mathrm{k}-8 \mathrm{k}$ & 0,05 & 0,5 \\
\hline $\mathrm{Cu}$ & $\begin{array}{l}\text { Daños hepáticos, enfermedad de Wilson, } \\
\text { insomnio }\end{array}$ & $175-250$ & 0,25 & 1 \\
\hline $\mathbf{N i}$ & $\begin{array}{l}\text { Dermatitis, náuseas, asma crónica, tos, } \\
\text { carcinógeno humano }\end{array}$ & -- & 0,2 & 0,5 \\
\hline $\mathbf{Z n}$ & $\begin{array}{l}\text { Depresión, letargo, signos neurológicos y aumento } \\
\text { de la sed }\end{array}$ & $6 \mathrm{k}$ & 0,8 & 3 \\
\hline $\mathbf{P b}$ & $\begin{array}{l}\text { Teratogenicidad cerebral, enfermedades renales, } \\
\text { vasculares y neuronales }\end{array}$ & $10 \mathrm{k}$ & 0,006 & $0,2-05$ \\
\hline $\mathrm{Hg}$ & $\begin{array}{l}\text { Artritis reumatoidea y enfermedades renales, } \\
\text { vasculares y neuronales }\end{array}$ & $150-300$ & 0,00003 & 0,02 \\
\hline
\end{tabular}

K: Significa miles de miligramos/día.

\section{Técnicas de tratamiento para remoción de metales pesados en aguas residuales}

La clasificación de las técnicas de tratamiento de metales pesados en las aguas residuales depende de diferentes factores, en este documento se han clasificado como convencionales, refiriéndose a las técnicas que habitualmente se emplean para la remoción de estos contaminantes y las no convencionales, aquellas que corresponden a procesos innovadores para la eliminación de metales en aguas residuales generalmente de origen industrial.

\subsection{Técnicas convencionales}

\subsubsection{Filtración por Membrana}

Esta tecnología presenta altas eficiencias, requiere poco espacio, no es selectiva y es de fácil operación, pero genera una gran cantidad de lodos que contienen metales. Se emplea en procesos para el tratamiento de agua potable, aguas residuales industriales y en menor medida aguas residuales domésticas. Las membranas pueden clasificarse de acuerdo a diferentes características como su peso molecular de corte, material de la membrana (sintéticos o polímeros naturales modificados, acoplados y estructurados), permeabilidad y solubilidad del soluto y el solvente en la película, superficie y espesor activo de la película, así como la carga de su superficie (Taylor \&Wiesner, 2002). La separación por membrana se emplea comúnmente para tratar y recuperar sales metálicas de residuos generados en procesos galvanoplásticos, en el reciclaje de aceites, en la producción alimentos y bebidas y en la explotación y producción de hidrocarburos (Nemerow \& Dasgupta, 1998; Ji, 2015).

2.1.1.1 Electrodiálisis. Es una técnica de descontaminación que puede remover componentes iónicos de soluciones acuosas empleando membranas permeables selectivas en un campo eléctrico constante (Guastalli, et al; 2004). Esta técnica tiene la capacidad de remover iones contaminantes cargados de hasta $0,0001 \mu \mathrm{m}$, mediante hojas o laminas porosas de resinas de intercambio iónico con una baja permeabilidad relativa para el agua (Taylor \& Wiesner, 2002). Tabla 3.

2.1.1.2 Osmosis Inversa. Es un proceso de permeación a través de membrana para la separación por difusión controlada o cribado. Tiene la capacidad de seleccionar elementos de tan solo $0.0001 \mathrm{~mm}$, lo que le otorga un amplio abanico de capacidades de tratamiento (Nemerow \& Dasgupta, 1998). En la tabla 4 se registran las condiciones observadas en estudios en que se emplea la osmosis inversa.

2.1.1.3 Nanofiltración. Es una técnica de tratamiento de agua relativamente reciente que utiliza membranas con poros muy pequeños $(<1 \mathrm{~nm})$ y requiere presiones de 
Tabla 3. Condiciones de la electrodiálisis en diferentes estudios como técnica para la eliminación de metales pesados.

\begin{tabular}{|c|c|c|c|c|c|c|c|}
\hline \multirow[b]{2}{*}{ Especie } & \multicolumn{5}{|c|}{ Condiciones } & \multirow{2}{*}{$\begin{array}{c}\% \\
\text { Eficiencia } \\
\text { de } \\
\text { remoción }\end{array}$} & \multirow[b]{2}{*}{ Ref. } \\
\hline & $\mathrm{pH}$ & $\begin{array}{l}\text { Concentración } \\
\text { Inicial }\end{array}$ & $\mathbf{t}_{\mathrm{Tr}}$ & $\begin{array}{c}\text { Temperatura } \\
{ }^{\circ} \mathrm{C}\end{array}$ & $\begin{array}{c}\text { Método y } \\
\text { complemento }\end{array}$ & & \\
\hline $\mathrm{Cu}^{+2}$ & 7 & $\begin{array}{l}0,12 \mathrm{~g} / \mathrm{L} ; 0,11 \\
\mathrm{~g} / \mathrm{L} ; 0,09 \mathrm{~g} / \mathrm{L}\end{array}$ & $2 \mathrm{~h}$ & 25 & $\begin{array}{l}\text { Electrodiálisis de } \\
\text { intercambio iónico } \\
\text { híbrido, con un DVB } \\
\text { entrecruzado grado } \\
\text { variable de 2, } 4 \text { y } 8 \% \text {. }\end{array}$ & 41,33 y 31 & $\begin{array}{l}\text { Mahuud, } \\
\text { et al; } 2012\end{array}$ \\
\hline $\mathrm{Co}^{+2}$ & 6 & $0,06-3,0 \mathrm{~g} / \mathrm{L}$ & $3 \mathrm{~h}$ & 25 & $\begin{array}{l}\text { Membrana Líquido } \\
\text { Hybrid de } 20 \text { - } 30 \mu \mathrm{m} \text { - } \\
\text { Electrodiálisis - } \\
\text { proceso de electrólisis. }\end{array}$ & $>75$ & $\begin{array}{l}\text { Sadyrbaeva, } \\
2014\end{array}$ \\
\hline $\mathrm{Cu}^{+2} \mathrm{Fe}^{+2}$ & NA & $9.0 \mathrm{~g} / \mathrm{L}, 0,5 \mathrm{~g} / \mathrm{L}$ & $\begin{array}{l}4 y \\
24 h\end{array}$ & 25 & $\begin{array}{l}\text { Electrodiálisis para la } \\
\text { separación de metales. } \\
\text { Compartimiento de } 5 \\
\text { celdas de } 14 \mathrm{~mm} \text { de } \\
\text { espesor. }\end{array}$ & 96,6 y 99,5 & $\begin{array}{l}\text { Cifuentes, } \\
\text { et al; } 2009\end{array}$ \\
\hline Mo & $3-8$ & $0,3 \mathrm{~g} / \mathrm{L}$ & $\begin{array}{c}1- \\
39 \\
\min \end{array}$ & 25 & $\begin{array}{l}\text { Separación de especies } \\
\text { de molibdeno por } \\
\text { electrodiálisis. }\end{array}$ & $45,2-66,6$ & $\begin{array}{l}\text { Cifuentes, } \\
\text { et al; } 2011\end{array}$ \\
\hline
\end{tabular}

$\mathrm{t}_{\mathrm{Tr}}:$ Tiempo de tratamiento

Tabla 4. Condiciones de la técnica de osmosis inversa en diferentes estudios.

\begin{tabular}{|c|c|c|c|c|c|c|c|}
\hline \multirow[b]{2}{*}{ Especie } & \multicolumn{5}{|c|}{ Condiciones } & \multirow{2}{*}{$\begin{array}{c}\% \% \\
\text { Eficiencia } \\
\text { de } \\
\text { remoción }\end{array}$} & \multirow[b]{2}{*}{ Ref. } \\
\hline & $\mathrm{pH}$ & $\begin{array}{l}\text { Concentración } \\
\text { Inicial }\end{array}$ & $\mathbf{t}_{\mathrm{Tr}}$ & $\begin{array}{c}\text { Temperatura } \\
{ }^{\circ} \mathrm{C}\end{array}$ & $\begin{array}{l}\text { Método y } \\
\text { complemento }\end{array}$ & & \\
\hline B & 9 & $\begin{array}{c}7000-15000 \\
\mathrm{mg} / \mathrm{L}\end{array}$ & NA & 25 & $\begin{array}{l}\text { Ósmosis inversa en } \\
\text { aplicaciones de } \\
\text { desalación de agua } \\
\text { de mar. Membrana } \\
\text { de } 20 \mu \mathrm{m}\end{array}$ & 25 & $\begin{array}{l}\text { Tu, et al; } \\
2012\end{array}$ \\
\hline $\mathrm{Fe}^{+2}$ & $\begin{array}{l}5,5- \\
8,5\end{array}$ & $20 \mathrm{mg} / \mathrm{L}$ & $4 \mathrm{~h}$ & $12-29$ & $\begin{array}{l}\text { Ósmosis inversa } \\
\text { (membrana AG2521TF } \\
10 \mu \mathrm{m} \text { ) y la oxidación } \\
\text { del concentrado por } \\
\text { energía solar foto- } \\
\text { Fenton }\end{array}$ & $30-40$ & $\begin{array}{c}\text { Ioannou, et } \\
\text { al; } 2013\end{array}$ \\
\hline $\mathrm{Ni}, \mathrm{Co}$ & 7 & $240 \mathrm{mg} / \mathrm{L}$ & NA & 25 & $\begin{array}{l}\text { Proceso de separación } \\
\text { por membrana Dow } \\
\text { Chemical. }\end{array}$ & 99,6 & $\begin{array}{c}\text { Guerrero, } \\
\quad \text { al } ; 2006\end{array}$ \\
\hline
\end{tabular}

$\mathrm{t}_{\mathrm{Tr}}:$ Tiempo de tratamiento. $\quad$ NA: No Aplica

funcionamiento en el rango de 10-50 bar. Por lo tanto, las membranas empleadas para la nanofiltración son capaces de retener especies neutras con peso molecular $<200$ $300 \mathrm{~g} / \mathrm{mol}$, y también para rechazar iones inorgánicos por un mecanismo de exclusión por tamaño en combinación con las interacciones electrostáticas entre los iones y la membrana cargada, presenta mayor rechazo de iones divalentes y menor rechazo de iones monovalentes, la presión de funcionamiento más baja, mayor flujo y menor consumo de energía en comparación con la osmosis inversa (González, et al; 2006). Estas características recomiendan la nanofiltración como una tecnología prometedora e innovadora que puede ser ampliamente aplicada en el agua potable y el tratamiento de efluentes industriales. En la tabla 5 se presentan tres estudios de remoción de metales pesados mediante esta técnica. 
2.1.1.4 Ultrafiltración. La ultrafiltración es un proceso de fraccionamiento selectivo utilizando presiones de hasta 145 psi (10 bares). La ultrafiltración se utiliza ampliamente en el fraccionamiento de leche y suero, $\mathrm{y}$ en fraccionamiento proteico. Concentra sólidos en suspensión y solutos de peso molecular mayor a 1000 umas. El permeado contiene solutos orgánicos de bajo peso molecular y sales (Alka, et al; 2012), (Tabla 6).

Tabla 5. Técnica de Nanofiltracion en diferentes estudios.

\begin{tabular}{|c|c|c|c|c|c|c|c|}
\hline \multirow[b]{2}{*}{ Especie } & \multicolumn{5}{|c|}{ Condiciones } & \multirow{2}{*}{$\begin{array}{c}\% \\
\text { Eficiencia } \\
\text { de } \\
\text { remoción }\end{array}$} & \multirow[b]{2}{*}{ Ref. } \\
\hline & $\mathbf{p H}$ & $\begin{array}{c}\text { Concentración } \\
\text { Inicial }\end{array}$ & $\mathbf{t}_{\mathbf{T r}}$ & $\begin{array}{c}\text { Temperatura } \\
{ }^{\circ} \mathrm{C}\end{array}$ & $\begin{array}{l}\text { Método y } \\
\text { complemento }\end{array}$ & & \\
\hline $\begin{array}{l}\mathrm{Pb}^{2+} \\
\mathrm{Cd}^{2+}\end{array}$ & 5,8 & $\begin{array}{c}150 \mathrm{mg} / \mathrm{L}, 40 \\
\mathrm{mg} / \mathrm{L}\end{array}$ & $10 \mathrm{~h}$ & 25 & $\begin{array}{l}\text { Nanofiltración, } \\
\text { recubrimiento de } \\
\text { poliamida aromática en } \\
\text { sustrato de polisulfona } \\
\text { con poros } 0,262 \mathrm{~nm} .\end{array}$ & $98,5-96$ & $\begin{array}{l}\text { Gherasim, } \\
\text { et al; } 2013\end{array}$ \\
\hline $\begin{array}{l}\mathrm{Fe}^{+2} \\
\mathrm{Al}^{2+} \\
\mathrm{Mg}^{2+}\end{array}$ & $6-7$ & $\begin{array}{l}4920 \mathrm{mg} / \mathrm{L} \text {, } \\
3050 \mathrm{mg} / \mathrm{L} \text {, } \\
3400 \mathrm{mg} / \mathrm{L}\end{array}$ & $36 \mathrm{~h}$ & 25 & $\begin{array}{l}\text { Purificación de ácido } \\
\text { fosfórico por nanofiltra- } \\
\text { tración. Membrana } \\
\text { DS5 DL } 0,5 \mathrm{~nm} \text {. }\end{array}$ & $98-96$ & $\begin{array}{l}\text { González, } \\
\text { et al; } 2006\end{array}$ \\
\hline $\begin{array}{l}\mathrm{Cd}^{+2} \\
\mathrm{Zn}^{+2}\end{array}$ & $\begin{array}{l}2- \\
11\end{array}$ & $80 \mathrm{mg} / \mathrm{L}$ & $\begin{array}{c}4- \\
24 h\end{array}$ & $20-22$ & $\begin{array}{l}\text { Tartrato de sodio y } \\
\text { potasio (PST) en un } \\
\text { proceso de nanofiltra- } \\
\text { ción con membrana GE } \\
\text { Osmosnic en poliamida } \\
\text { aromática. }\end{array}$ & $95,5-98$ & $\begin{array}{l}\text { Liu, et al; } \\
\quad 2012\end{array}$ \\
\hline $\mathrm{Mg}^{2+} \mathrm{Li}^{+}$ & $6-7$ & $\begin{array}{c}2000,8000 \\
\mathrm{mg} / \mathrm{L}\end{array}$ & NA & 20 & $\begin{array}{l}\text { Recuperación de } \mathrm{Li} \\
\text { en alta relación de } \\
\text { salmuera con } \\
\text { membrana NF } 90 \text {. }\end{array}$ & $80,1-85$ & $\begin{array}{l}\mathrm{Bi}, \text { et al; } \\
2014\end{array}$ \\
\hline
\end{tabular}

$\mathrm{t}_{\mathrm{Tr}}:$ Tiempo de tratamiento

Tabla 6. Ultrafiltración en diferentes estudios para remover metales pesados.

\begin{tabular}{|c|c|c|c|c|c|c|c|}
\hline \multirow[b]{2}{*}{ Especie } & \multicolumn{5}{|c|}{ Condiciones } & \multirow{2}{*}{$\begin{array}{c}\% \\
\text { Eficiencia } \\
\text { de } \\
\text { remoción } \\
\end{array}$} & \multirow[b]{2}{*}{ Ref. } \\
\hline & $\mathrm{pH}$ & $\begin{array}{c}\text { Concentración } \\
\text { Inicial }\end{array}$ & $\mathbf{t}_{\mathrm{Tr}}$ & $\begin{array}{c}\text { Temperatura } \\
{ }^{\circ} \mathrm{C}\end{array}$ & $\begin{array}{c}\text { Método y } \\
\text { complemento }\end{array}$ & & \\
\hline $\begin{array}{c}\mathrm{Cd}^{2+}, \mathrm{Fe}^{2+}, \\
\mathrm{Cu}^{2+}, \mathrm{Zn}^{+2}, \\
\mathrm{Ni}^{2+}\end{array}$ & 6,5 & $20 \mathrm{mM}$ & $\begin{array}{c}2,5 \\
h\end{array}$ & Ambiente & $\begin{array}{l}\text { Nanofiltración micelar } \\
\text { mejorada con nonaoxie- } \\
\text { tileno oleiléter RO90 } \\
\text { en contraste con dodecil- } \\
\text { sulfato de sodio (SDS). }\end{array}$ & $>95$ & $\begin{array}{l}\text { Schwarze, } \\
\text { et al; } 2015\end{array}$ \\
\hline $\mathrm{Cd}^{2+}, \mathrm{Cu}^{2+}$ & $\begin{array}{c}3,5- \\
4,5\end{array}$ & $\begin{array}{c}0,4-0,7 \mathrm{mg} / \mathrm{L}, \\
0,4-0,6 \mathrm{mg} / \mathrm{L}\end{array}$ & NA & 25 & $\begin{array}{l}\text { Separación de } \mathrm{Cd} \text { y } \mathrm{Cu} \\
\text { de aguas ricas en } \\
\text { fosforo por } \\
\text { ultrafiltración micelar } \\
\text { mejorada, membrana } \\
\text { Amicon } 8400 \text { stirred } \\
\text { cell, Millipore. }\end{array}$ & 84,3 y 75,0 & $\begin{array}{l}\text { Landaburu, } \\
\text { et al; } 2011\end{array}$ \\
\hline $\mathrm{Cd}^{2+}$ & $\begin{array}{l}2- \\
13\end{array}$ & $50 \mathrm{mg} / \mathrm{L}$ & NA & $5-45$ & $\begin{array}{l}\text { Evaluación de micelar } \\
\text { mejorada ultrafiltración } \\
\text { con agentes } \\
\text { tensoactivos mixtos. }\end{array}$ & 98 & $\begin{array}{c}\text { Huang, et al; } \\
2014\end{array}$ \\
\hline
\end{tabular}

$\mathrm{t}_{\mathrm{Tr}}:$ Tiempo de tratamiento 
2.1.2 Intercambio Iónico. es el proceso a través del cual los iones en solución se transfieren a una matriz sólida que, a su vez liberan iones de un tipo diferente, pero de la misma carga. El intercambio iónico es un proceso de separación física en la que los iones intercambiados no se modifiquen químicamente. Las principales ventajas de intercambio iónico son la recuperación del valor del metal, la selectividad, menos volumen de lodos producidos y la reunión de las especificaciones de descarga estrictas (Zewail \& Yousef, 2015). En la tabla 7 se exponen las condiciones de tres estudios mediante este método.

\subsubsection{Adsorción (Convencional).}

La técnica presenta remoción de una amplia variedad de contaminantes, alta capacidad, cinética rápida y posiblemente selectiva dependiendo de adsorbente de lo cual también depende su rendimiento, básicamente por la estructura física del mismo (Liu \& Lee, 2014). Los carbones activados, arcillas, biopolímeros, Zeolitas, perlas de sílice y plantas o desechos lignocelulósicos son algunos de los adsorbentes, generalmente con procesos variados de modificación química (Wan Ngah, et al; 2011), comúnmente empleados para remover colorantes iónicos, metales pesados, materiales radioactivos entre otros contaminantes orgánicos e inorgánicos generados por diferentes tipos de industrias (Osei Boamah, et al; 2015).
2.1.3.1 Carbón Activado. ha demostrado ser un adsorbente eficiente para la eliminación de una amplia variedad de contaminantes orgánicos e inorgánicos presentes en el medio ambiente acuático. Debido a sus áreas superficiales porosas que van desde 500 hasta $1.500 \mathrm{~m}^{2} /$ g, así como la presencia de un amplio espectro de superficie funcional que la hace accesible a diferentes reactivos (Karnib, et al; 2014). En la tabla 8 se exponen las condiciones de tres estudios mediante este método.

2.1.3.2 Nanotubos de carbono. Han despertado una amplia atención como un nuevo tipo de adsorbentes debido a su capacidad excepcional para la eliminación de diversos contaminantes inorgánicos y orgánicos, y radionúcleidos a partir de grandes volúmenes de aguas residuales. Esta revisión resume (Tabla 9) algunas condiciones en que se han empleado los nanotubos de carbono y sus propiedades relacionadas con la adsorción de diversos metales pesados en soluciones acuosas (Ren, et al; 2011).

\subsubsection{Precipitación química}

Es la técnica más utilizada en los procesos industriales ya que es relativamente sencilla de operar, económica y selectiva, aunque su mantenimiento si es costoso debido a la alta generación de lodos. En la Tabla 10 se presentan rendimientos de tratamientos como precipitación de hidróxido, técnica de bajo costo, fácil control del pH y eliminación por floculación y sedimentación (Huisman, et al; 2006). Igualmente se

Tabla 7. Condiciones experimentales de intercambio iónico a través de resinas.

\begin{tabular}{|c|c|c|c|c|c|c|c|}
\hline \multirow[b]{2}{*}{ Especie } & \multicolumn{5}{|c|}{ Condiciones } & \multirow{2}{*}{$\begin{array}{c}\% \\
\text { Eficiencia } \\
\text { de } \\
\text { remoción }\end{array}$} & \multirow[b]{2}{*}{ Ref. } \\
\hline & pH & $\begin{array}{c}\text { Concentración } \\
\text { Inicial }\end{array}$ & $\mathrm{tTr}$ & $\begin{array}{c}\text { Temperatura } \\
{ }^{\circ} \mathrm{C}\end{array}$ & $\begin{array}{c}\text { Método y } \\
\text { Complemento }\end{array}$ & & \\
\hline $\begin{array}{l}\mathrm{Fe}^{2+}, \\
\mathrm{Cu}^{2+}, \\
\mathrm{Zn}^{2+}, \\
\mathrm{Cd}^{2+}, \\
\mathrm{Pb}^{2+}\end{array}$ & 4,5 & $24-58 \mathrm{mg} / \mathrm{L}$ & $4 \mathrm{~h}$ & 25 & $\begin{array}{l}\text { Resina } \\
\text { THQSA sintetizada por } \\
\text { reacción de epoxi propil } \\
\text { éter de tamarindo con } \\
\text { ácido 8- } \\
\text { hidroxiquinolina-5- } \\
\text { sulfónico }\end{array}$ & $60-90$ & $\begin{array}{c}\text { Chahar \& } \\
\text { Singh, } 2014 .\end{array}$ \\
\hline $\mathrm{Cd}^{2+}$ & 3 & $30 \mathrm{mg} / \mathrm{L}$ & $24 \mathrm{~h}$ & 26 & $\begin{array}{l}\text { Adsorción y desorción } \\
\text { de Cd (II) con resina } \\
\text { D001. }\end{array}$ & $185,8 \mathrm{mg} / \mathrm{g}$ & $\begin{array}{l}\text { Zhen, et al; } \\
2015\end{array}$ \\
\hline $\begin{array}{l}\mathrm{Cr}^{3+}, \mathrm{Ni}^{2+} \\
\mathrm{Cu}^{2+}, \mathrm{Cd}^{2+} \\
\mathrm{Co}^{2+}\end{array}$ & $5-6$ & $0,5-20 \mathrm{mM}$ & $24 \mathrm{~h}$ & 26 & $\begin{array}{l}\text { Resina sintetizado en la } \\
\text { reacción de poli metil } \\
\text { vinil éter alt anhídrido } \\
\text { maleico (MVE-alt-MA), } \\
\text { polímero con una base } \\
\text { de Schiff. }\end{array}$ & $\begin{array}{c}\text { En un rango } \\
\text { de } 29,95- \\
157,25 \\
\mathrm{mg} / \mathrm{g}\end{array}$ & $\begin{array}{l}\text { Cegłowski } \\
\text { \& Schroeder, } \\
2015\end{array}$ \\
\hline
\end{tabular}

$\mathrm{t}_{\mathrm{Tr}}$ : Tiempo de tratamiento 
Tabla 8. Condiciones experimentales de algunos carbones.

\begin{tabular}{|c|c|c|c|c|c|c|c|}
\hline \multirow[b]{2}{*}{ Especie } & \multicolumn{5}{|c|}{ Condiciones } & \multirow{2}{*}{$\begin{array}{c}\% \\
\text { Eficiencia } \\
\text { de } \\
\text { remoción }\end{array}$} & \multirow[b]{2}{*}{ Ref. } \\
\hline & pH & $\begin{array}{c}\text { Concentración } \\
\text { Inicial }\end{array}$ & $\mathrm{tTr}$ & $\begin{array}{c}\text { Temperatura } \\
{ }^{\circ} \mathrm{C}\end{array}$ & pH & & \\
\hline $\begin{array}{l}\mathrm{Pb}^{2+}, \mathrm{Zn}^{2+}, \\
\mathrm{Cu}^{2+}, \mathrm{Cd}^{2+}\end{array}$ & 5 & $10-180 \mathrm{mg} / \mathrm{L}$ & $24 \mathrm{~h}$ & 20 & $\begin{array}{l}\text { Carbono mesoporoso } \\
\text { ordenado CMK-3. }\end{array}$ & $\begin{array}{c}\text { Desde } 29 \\
\mathrm{mg} / \mathrm{g} \text { de } \\
\mathrm{Zn}^{2+} \text { a } 177 \\
\mathrm{mg} / \mathrm{g} \text { de } \\
\mathrm{Pb}^{2+}\end{array}$ & $\begin{array}{l}\text { Barczak, } \\
\text { et al; } 2015\end{array}$ \\
\hline $\mathrm{Cr}$ & 7,8 & $0,12 \mathrm{mg} / \mathrm{L}$ & $2 \mathrm{~min}$ & Ambiente & $\begin{array}{l}\text { Fe-BC (hierro a base de } \\
\text { carbón de bambú) }\end{array}$ & $>0.05 \mathrm{mg} / \mathrm{L}$ & $\begin{array}{l}\text { Wang, et al; } \\
2011\end{array}$ \\
\hline $\mathrm{Pb}^{2+}$ & 4 & $100 \mathrm{mg} / \mathrm{L}$ & $6 \mathrm{~h}$ & Ambiente & $\begin{array}{l}\text { Carbón activado } \\
\text { procedente de huesos de } \\
\text { vaca. }\end{array}$ & $50 \%$ & $\begin{array}{l}\text { Prado, et al; } \\
\quad 2014\end{array}$ \\
\hline $\begin{array}{l}\mathrm{Cu}^{2+} \\
\mathrm{Ni}^{2+} \\
\mathrm{Zn}^{2+}\end{array}$ & 5,5 & $10-100 \mathrm{mg} / \mathrm{L}$ & $\begin{array}{l}5 \min \\
-20 h\end{array}$ & $20-40$ & $\begin{array}{l}\text { Carbón activado } \\
\text { producido a partir de } \\
\text { piedras tunecinas. }\end{array}$ & $\begin{array}{c}18,68 \mathrm{mg} / \mathrm{g} \\
\mathrm{de} \mathrm{Cu} \\
16,12 \mathrm{mg} / \mathrm{g} \\
\text { de } \mathrm{Ni} \mathrm{y} \\
12,19 \mathrm{mg} / \mathrm{g} \\
\text { de } \mathrm{Zn}\end{array}$ & $\begin{array}{l}\text { Bouhamed, } \\
\text { et al; } 2015\end{array}$ \\
\hline
\end{tabular}

$\mathrm{t}_{\mathrm{Tr}}:$ Tiempo de tratamiento

Tabla 9. Condiciones experimentales de nanotubo de carbón con otros complementos.

\begin{tabular}{|c|c|c|c|c|c|c|c|}
\hline \multirow[b]{2}{*}{ Especie } & \multicolumn{5}{|c|}{ Condiciones } & \multirow{2}{*}{$\begin{array}{c}\% \\
\text { Eficiencia } \\
\text { de } \\
\text { remoción }\end{array}$} & \multirow[b]{2}{*}{ Ref. } \\
\hline & pH & $\begin{array}{c}\text { Concentración } \\
\text { Inicial }\end{array}$ & $\mathbf{t} \mathbf{T r}$ & $\begin{array}{c}\text { Temperatura } \\
{ }^{\circ} \mathrm{C}\end{array}$ & pH & & \\
\hline $\begin{array}{l}\mathrm{Co}^{2+}, \mathrm{Ca}^{2} \\
+, \mathrm{Mg}^{2+} \\
\mathrm{Zn}^{2+}, \mathrm{Fe}^{3}\end{array}$ & 6 & $\begin{array}{c}0,8 \mathrm{mg} / \mathrm{L}, \\
40 \mathrm{mg} / \mathrm{L}, 23 \mathrm{mg} / \mathrm{L}, \\
0,2 \mathrm{mg} / \mathrm{L}, \\
0,32 \mathrm{mg} / \mathrm{L} \\
\text { respectivamente }\end{array}$ & $\begin{array}{c}10 \\
\min \end{array}$ & Ambiente & $\begin{array}{l}\text { Diatomita tierra } \\
\text { compuesto por el } \\
\text { método de deposición } \\
\text { de vapor químico }\end{array}$ & 91,3 & $\begin{array}{c}\text { Alijani, et al; } \\
2014\end{array}$ \\
\hline $\mathrm{Zn}^{2+}$ & 10 & $1,1 \mathrm{mg} / \mathrm{L}$ & $\begin{array}{l}120 \\
\min \end{array}$ & Ambiente & $\begin{array}{l}\text { NCT (nanotubos de } \\
\text { carbón funcionalizado) } \\
\text { y BM (biocarbon } \\
\text { magnética) }\end{array}$ & $\begin{array}{l}99 \text { NCT y } \\
75 \mathrm{BM}\end{array}$ & $\begin{array}{l}\text { Mubarak, } \\
\text { et al; } 2013\end{array}$ \\
\hline $\mathrm{Zn}, \mathrm{Cu}, \mathrm{Ni}$ & 5 & $\begin{array}{c}60 \mathrm{mg} / \mathrm{L} \mathrm{Zn}, 50 \\
\mathrm{mg} / \mathrm{L} \mathrm{Cu}, 40 \\
\mathrm{mg} / \mathrm{L} \mathrm{Ni}\end{array}$ & & Ambiente & $\begin{array}{l}\text { Nanotubos de carbono } \\
\text { de pared múltiple } \\
\text { (MWCNT) impregnado } \\
\text { con di-(2-etil hexilo } \\
\text { ácido fosfórico) } \\
\text { (D2EHPA) y óxido de } \\
\text { fosfina de tri-n-octilo } \\
\text { (TOPO). }\end{array}$ & $\begin{array}{c}\text { Capacidad } \\
\text { de } \\
\text { absorción } \\
4,9 \mathrm{mg} / \mathrm{g} \\
\mathrm{Cu} \\
4,78 \mathrm{mg} / \mathrm{g} \\
\mathrm{Ni}, 4,8 \mathrm{mg} / \mathrm{g} \\
\mathrm{Zn}\end{array}$ & $\begin{array}{l}\text { Palanivelu, } \\
\text { et al; } 2011\end{array}$ \\
\hline
\end{tabular}

$\mathrm{t}_{\mathrm{Tr}}:$ Tiempo de tratamiento.

emplea la precipitación por sulfuros aunque con solubilidades bajas y precipitados no anfóteros, por lo que puede lograr altos rendimientos; también se han empleado sustancias quelantes aunque presentan desventajas considerables como la carencia de uniones necesarias y demasiados riesgos ambientales (Chen, et al; 2009).
2.1.5 Electrocoagulación. Es un proceso que aplica los principios de la coagulación-floculación en un reactor electrolítico. Este es un recipiente dotado de una fuente de corriente y varios electrodos encargados de aportar los iones desestabilizadores de partículas coloidales que reemplazan las funciones de los compuestos químicos que se utilizan en el tratamiento 
convencional, induciendo la corriente eléctrica en el agua a través de placas metálicas paralelas de hierro o aluminio (Morante, 2002) como en estos tres casos expuestos en la tabla 11.
2.1.6 Coagulación-Floculación. Es el método mediante el cual se logra desestabilizar el coloide y aglomerar posteriormente. En la primera etapa la coagulación elimina la doble capa eléctrica que

Tabla 10. Remociones de metales pesados empleando técnicas de precipitación química.

\begin{tabular}{|c|c|c|c|c|c|c|c|}
\hline \multirow[b]{2}{*}{ Especie } & \multicolumn{5}{|c|}{ Condiciones } & \multirow{2}{*}{$\begin{array}{c}\% \\
\text { Eficiencia } \\
\text { de } \\
\text { remoción }\end{array}$} & \multirow[b]{2}{*}{ Ref. } \\
\hline & pH & $\begin{array}{l}\text { Concentración } \\
\text { Inicial }\end{array}$ & $\mathrm{tTr}$ & $\begin{array}{c}\text { Temperatura } \\
{ }^{\circ} \mathrm{C}\end{array}$ & $\begin{array}{l}\text { Método y } \\
\text { complemento }\end{array}$ & & \\
\hline $\mathrm{Ni}, \mathrm{Zn}, \mathrm{Al}$ & 12 & $22 \mathrm{mg} / \mathrm{L}$ & $1 \mathrm{~h}$ & Ambiente & $\begin{array}{l}\text { Precipitación por } \\
\text { galvanoplastia } \mathrm{Ni} \\
\text { servoasistida por } \mathrm{LDH} \text {. }\end{array}$ & $80-90$ & $\begin{array}{l}\mathrm{Xu}, \text { et al; } \\
2015\end{array}$ \\
\hline $\mathrm{Hg} 2+$ & $\begin{array}{c}4,7- \\
6,4\end{array}$ & $65,6 \mu \mathrm{g} / \mathrm{L}$ & $1 \mathrm{~h}$ & Ambiente & $\begin{array}{l}\text { Precipitación con 1,3- } \\
\text { benzenediamidoethanet } \\
\text { hiolate }\end{array}$ & $>99.9$ & $\begin{array}{l}\text { Blue, et al; } \\
2008\end{array}$ \\
\hline $\begin{array}{c}\mathrm{As}^{2+}, \\
\mathrm{Cu}^{2+}, \\
\mathrm{Cd}^{2+}, \mathrm{Pb}^{2+}\end{array}$ & 1 & $\begin{array}{c}\text { As } 6,080 \mathrm{mg} / \mathrm{L}, \\
\mathrm{Cd} 95 \mathrm{mg} / \mathrm{L}, \mathrm{Cu} \\
70 \mathrm{mg} / \mathrm{L} \mathrm{y} \mathrm{Pb} 45 \\
\mathrm{mg} / \mathrm{L}\end{array}$ & $1 \mathrm{~h}$ & Ambiente & $\begin{array}{l}\text { Reactor con distribuidor } \\
\text { multiarranque } \\
\text { sumergido para la } \\
\text { alimentación de sulfuro. }\end{array}$ & $>99.9$ & $\begin{array}{l}\text { Gou, et al; } \\
2015\end{array}$ \\
\hline $\begin{array}{l}\mathrm{Cd}, \mathrm{Cr}, \\
\mathrm{Cu}, \mathrm{Pb}, \\
\mathrm{Zn}, \mathrm{As}, \\
\mathrm{Hg}\end{array}$ & 4 & $1 \mathrm{mg} / \mathrm{L}$ & $5 \mathrm{~min}$ & Ambiente & $\begin{array}{l}\text { Nanocompuestos como } \\
\text { Micropartículas } \\
\text { superparamagnéticas } \\
\text { que actúan como } \\
\text { semillas para } \\
\text { precipitados de } \\
\text { hidróxido de hierro. }\end{array}$ & $95-99$ & $\begin{array}{l}\text { Mandel, et al; } \\
2013\end{array}$ \\
\hline
\end{tabular}

$\mathrm{t}_{\mathrm{Tr}}:$ Tiempo de tratamiento

Tabla 11. Condiciones experimentales para remover metales pesados mediante electrocoagulación.

\begin{tabular}{|c|c|c|c|c|c|c|c|}
\hline \multirow[b]{2}{*}{ Especie } & \multicolumn{5}{|c|}{ Condiciones } & \multirow{2}{*}{$\begin{array}{c}\% \\
\text { Eficiencia } \\
\text { de } \\
\text { remoción }\end{array}$} & \multirow[b]{2}{*}{ Ref. } \\
\hline & pH & $\begin{array}{c}\text { Concentración } \\
\text { Inicial }\end{array}$ & $t \operatorname{Tr}$ & $\begin{array}{c}\text { Temperatura } \\
{ }^{\circ} \mathrm{C}\end{array}$ & $\begin{array}{c}\text { Método y } \\
\text { Complemento }\end{array}$ & & \\
\hline $\mathrm{Cu}^{2+}$ & $4-8$ & $12 \mathrm{ppm}$ & $\begin{array}{c}30 \\
\min \end{array}$ & Ambiente & $\begin{array}{l}\text { Después de la } \\
\text { electroquímica, los } \\
\text { líquidos dosificados se } \\
\text { llevaron a floculación y } \\
\text { sedimentación }\end{array}$ & $95 \%$ & $\begin{array}{l}\text { Jack, et al; } \\
2013\end{array}$ \\
\hline $\begin{array}{l}\mathrm{Ni}^{2+}, \mathrm{Pb}^{2+}, \\
\mathrm{Cd}^{2+}\end{array}$ & $1-7$ & $1 \mathrm{ppm}$ & $\begin{array}{l}0-10 \\
\min \end{array}$ & Ambiente & $\begin{array}{l}\text { Potasio clorhídrico fue } \\
\text { adicionado para } \\
\text { incrementar la } \\
\text { conductividad de la } \\
\text { solución }\end{array}$ & $\begin{array}{c}99,0 \%, \\
98,3 \%, \\
96,8 \% \\
\text { respectivam } \\
\text { ente, } \\
\text { utilizando } \\
\text { electrodos } \\
\text { de hierro } \\
\end{array}$ & $\begin{array}{c}\text { Khosa, et al; } \\
2013\end{array}$ \\
\hline $\begin{array}{l}\mathrm{Cu}^{2+} \\
\mathrm{Cr}^{3+} \\
\mathrm{Ni}^{2+} \\
\mathrm{Zn}^{2+}\end{array}$ & 9,5 & $\begin{array}{c}93,33,57,20 \\
\text { ppm } \\
\text { respectivamente }\end{array}$ & $45 \mathrm{~min}$ & 26 & $\begin{array}{l}\text { Un electro-reactor con } \\
\text { electrodos de acero de } \\
\text { carbono de } \\
\text { configuraciones } \\
\text { monopolares }(6)\end{array}$ & $\begin{array}{l}>97 \text { en } \\
\text { todos los } \\
\text { metales }\end{array}$ & $\begin{array}{l}\text { Al-Shannag, } \\
\text { et al; } 2015\end{array}$ \\
\hline
\end{tabular}

$\mathrm{t}_{\mathrm{Tr}}:$ Tiempo de tratamiento. 
caracteriza a los coloides y con la floculación se aglomeran los coloides mediante la atracción de partículas con el aglutinamiento de los floculantes. Los factores más importantes que se deben tener en cuenta para esta técnica son la dosis química apropiada, el efecto energético de la mezcla y el tiempo de la mezcla; los coagulantes químicos que se utilizan comúnmente en el tratamiento de aguas residuales incluyen alumbre $\left(\mathrm{Al}_{2}\left(\mathrm{SO}_{4}\right)_{3} \cdot 18 \mathrm{H}_{2} \mathrm{O}\right)$, cloruro férrico $\left(\mathrm{FeCl}_{3} \cdot 6 \mathrm{H}_{2} \mathrm{O}\right)$, sulfato férrico $\left(\mathrm{Fe}_{2}\left(\mathrm{SO}_{4}\right)_{3}\right)$, sulfato ferroso $\left(\mathrm{FeSO}_{4} \cdot 7 \mathrm{H}_{2} \mathrm{O}\right)$ y cal $\left(\mathrm{Ca}(\mathrm{OH})_{2}\right)$ (Ismail, et al; 2012). Se presentan dos casos en la tabla 12.

\subsubsection{Electrofloculación. Es un proceso químico con} adición electrolítica de iones metálicos. Su eficiencia en la remoción de los contaminantes se debe a que son arrastrados por las burbujas de gas $\left(\mathrm{H}_{2} \mathrm{y} \mathrm{O}_{2}\right)$ que se producen en el sistema por lo que salen a flote en la superficie (Tabla 13).
2.1.8 Flotación. El proceso de flotación ion se basa en impartir las especies iónicas de metal en las aguas residuales hidrófobas mediante el uso de agentes activos de superficie (tensoactivos) y la posterior eliminación de estas especies hidrófobas por burbujas de aire (Polat \& Erdogan, 2007). En la Tabla 14 se observan dos casos experimentales de la técnica de flotación.

2.1.9 Fotocatálisis en la degradación de metales pesados. Se basa en la transferencia de carga a través de la interfaz entre el semiconductor y la solución acuosa contaminada. Donde la conductividad aumenta con la temperatura, y se genera un par de electrón-hueco, ocurriendo la adsorción de los fotones y la distribución de diferentes estados electrónicos en la superficie, degradando moléculas orgánicas colorantes y metales pesados. (Paez \& Taborda, 2006). En la tabla 15 se exponen estudios

Tabla 12. Remoción de metales pesados mediante Coagulación-Floculación

\begin{tabular}{|c|c|c|c|c|c|c|c|}
\hline \multirow[b]{2}{*}{ Especie } & \multicolumn{5}{|c|}{ Condiciones } & \multirow{2}{*}{$\begin{array}{c}\% \\
\text { Eficiencia } \\
\text { de } \\
\text { remoción }\end{array}$} & \multirow[b]{2}{*}{ Ref. } \\
\hline & $\mathbf{p H}$ & $\begin{array}{c}\text { Concentración } \\
\text { Inicial }\end{array}$ & tTr & $\begin{array}{c}\text { Temperatura } \\
{ }^{\circ} \mathrm{C}\end{array}$ & pH & & \\
\hline $\mathrm{Fe}, \mathrm{Zn}, \mathrm{Ni}$ & 7 & $\begin{array}{c}\mathrm{Fe} 15,2 \mathrm{mg} / \mathrm{L} ; \mathrm{Zn} \\
5,8 \mathrm{mg} / \mathrm{L} ; \mathrm{Ni} 6,7 \\
\mathrm{mg} / \mathrm{L}\end{array}$ & $30 \mathrm{~min}$ & Ambiente & $\begin{array}{l}\text { Se utilizaron tres } \\
\text { coagulantes (alumbre, } \\
\text { cloruro férrico y cloruro } \\
\text { de polialuminio) y } \\
\text { poliacrilamida aniónico } \\
\text { como floculante }\end{array}$ & $\begin{array}{l}98,83,63, \\
\text { respectiva } \\
\text { mente }\end{array}$ & $\begin{array}{c}\text { Bakar, et al; } \\
2013\end{array}$ \\
\hline $\mathrm{Mg}, \mathrm{Fe}$ & $8-9$ & $\begin{array}{c}308,7 \mathrm{mg} / \mathrm{L}, \\
145,6 \mathrm{mg} / \mathrm{L}, \\
\text { respectivamente }\end{array}$ & $\begin{array}{l}2-15 \\
\min \end{array}$ & 28 & $\begin{array}{l}\text { Se utiliza como } \\
\text { coagulante } \mathrm{Ca}(\mathrm{OH})_{2}\end{array}$ & $\begin{array}{l}91,3 \text { y } 100 \\
\text { respectiva } \\
\text { mente }\end{array}$ & $\begin{array}{c}\text { Carrera, et al; } \\
2012\end{array}$ \\
\hline
\end{tabular}

$\mathrm{t}_{\mathrm{Tr}}:$ Tiempo de tratamiento

Tabla 13. Remoción de metales pesados mediante Electrofloculación.

\begin{tabular}{|c|c|c|c|c|c|c|c|}
\hline \multirow[b]{2}{*}{ Especie } & \multicolumn{5}{|c|}{ Condiciones } & \multirow{2}{*}{\begin{tabular}{|c|}
$\%$ \\
Eficiencia \\
de \\
remoción
\end{tabular}} & \multirow[b]{2}{*}{ Ref. } \\
\hline & pH & $\begin{array}{c}\text { Concentración } \\
\text { Inicial }\end{array}$ & $\mathrm{tTr}$ & $\begin{array}{c}\text { Temperatura } \\
{ }^{\circ} \mathrm{C}\end{array}$ & pH & & \\
\hline $\begin{array}{c}\mathrm{Pb}, \mathrm{Ni}, \mathrm{Cd}, \\
\mathrm{Co}\end{array}$ & - & $\begin{array}{c}\text { Salinidad de } 0,4 \\
\text { a } 1,2 \%\end{array}$ & - & Ambiente & Electrofloculación & $\begin{array}{c}94,4 ; 92,8 ; \\
64,4 ; 35.5 \\
\text { respectivam } \\
\text { ente }\end{array}$ & $\begin{array}{c}\text { Chenar, et al; } \\
2013\end{array}$ \\
\hline $\begin{array}{c}\mathrm{Cd}, \mathrm{Zn}, \mathrm{Pb} \\
\text { y } \mathrm{Cu}\end{array}$ & 11 & $1 \%$ & $30 \mathrm{~min}$ & Ambiente & Electrofloculación $2 \mathrm{v}$ & $\begin{array}{c}100 ; 99 ; \\
95,5 ; 98,6 \\
\text { para } \mathrm{Cd}, \\
\mathrm{Zn}, \mathrm{Pb} \text { y } \mathrm{Cu} \\
\text { respectivam } \\
\text { ente. }\end{array}$ & $\begin{array}{l}\text { Fan, et al; } \\
\quad 2012\end{array}$ \\
\hline $\mathrm{Cd}$ & 7,3 & $0,02 \%$ & $5 \mathrm{~min}$ & 50 & $\begin{array}{c}\text { Electrofloculación } \\
\text { monopolar }\end{array}$ & 100 & $\begin{array}{l}\text { Khaled, et al; } \\
2015 .\end{array}$ \\
\hline
\end{tabular}

$\mathrm{t}_{\mathrm{Tr}}:$ Tiempo de tratamiento 
Tabla 14. Remoción de metales pesados mediante la técnica de flotación.

\begin{tabular}{|c|c|c|c|c|c|c|c|}
\hline \multirow[b]{2}{*}{ Especie } & \multicolumn{5}{|c|}{ Condiciones } & \multirow{2}{*}{$\begin{array}{c}\% \\
\text { Eficiencia } \\
\text { de } \\
\text { remoción }\end{array}$} & \multirow[b]{2}{*}{ Ref. } \\
\hline & $\mathbf{p H}$ & $\begin{array}{c}\text { Concentración } \\
\text { Inicial }\end{array}$ & tTr & $\begin{array}{l}\text { Temperatura } \\
{ }^{\circ} \mathrm{C}\end{array}$ & pH & & \\
\hline $\mathrm{Cu}^{2+}$ & & $1763 \mathrm{mg} / \mathrm{kg}$ & $2 \mathrm{~h}$ & Ambiente & $\begin{array}{l}\text { Flotación en sedimentos } \\
\text { degradados. }\end{array}$ & $60,1-70,4$ & $\begin{array}{c}\text { Palakkeel, et } \\
\text { al; } 2012\end{array}$ \\
\hline $\begin{array}{c}\mathrm{Fe}, \mathrm{Cu}, \mathrm{Ni}, \\
\mathrm{Mn}, \mathrm{Cd} \\
\mathrm{Pb}, \mathrm{Zn}\end{array}$ & $\begin{array}{c}1 \mathrm{a} \\
3 ; \\
10 \mathrm{a} \\
12\end{array}$ & $\begin{array}{c}8,542 \mathrm{mg} / \mathrm{L} ; \\
0,063 \mathrm{mg} / \mathrm{L} ; \\
0,349 \mathrm{mg} / \mathrm{L} ; \\
0,142 \mathrm{mg} / \mathrm{L} ; \\
0,00138 \mathrm{mg} / \mathrm{L} ; \\
0,025 \mathrm{mg} / \mathrm{L} ; \\
0,599 \mathrm{mg} / \mathrm{L}\end{array}$ & $2 \mathrm{~h}$ & Ambiente & $\begin{array}{l}\text { Ensayos de flotación } \\
\text { por aire disuelto (FAD) } \\
\text { con } 300 \mathrm{mg} / \mathrm{l} \text { de cloruro } \\
\text { férrico como coagulante } \\
\text { y } 3 \text { valores de pH ácidos } \\
\text { y } 3 \text { alcalinos }\end{array}$ & $\begin{array}{c}37,4 ; 43,2 ; \\
25,7 ; 24,3 ; \\
84,6 ; 90,1 ; \\
52,1\end{array}$ & $\begin{array}{c}\text { Méndez, et } \\
\text { al; } 2008\end{array}$ \\
\hline
\end{tabular}

$\mathrm{t}_{\mathrm{Tr}}:$ Tiempo de tratamiento

Tabla 15. Fotocatálisis como método para la eliminación de metales pesados.

\begin{tabular}{|c|c|c|c|c|c|c|c|}
\hline \multirow[b]{2}{*}{ Especie } & \multicolumn{5}{|c|}{ Condiciones } & \multirow{2}{*}{$\begin{array}{c}\% \\
\text { Eficiencia } \\
\text { de } \\
\text { remoción }\end{array}$} & \multirow[b]{2}{*}{ Ref. } \\
\hline & pH & $\begin{array}{l}\text { Concentra- } \\
\text { ción Inicial }\end{array}$ & tTr & $\begin{array}{c}\text { Temperatura } \\
{ }^{\circ} \mathrm{C}\end{array}$ & pH & & \\
\hline $\begin{array}{l}\mathrm{Cr}^{6+} ; \mathrm{Cr}^{3+} ; \\
\mathrm{Cr} \text { (total) }\end{array}$ & 5 & $10 \mathrm{mg} / \mathrm{L}$ & $\begin{array}{c}90 \\
\min \end{array}$ & 20 a 75 & $\begin{array}{l}\text { Sinergia de la } \\
\text { fotocatálisis y de } \\
\text { adsorción para la } \\
\text { eliminación simultánea } \\
\text { de } \mathrm{Cr}^{6+} \text { y } \mathrm{Cr}^{3+} \text { con el } \\
\mathrm{TiO}_{2} \text { y nanotubos de } \\
\text { titanato. }\end{array}$ & $\begin{array}{l}98,38 ; \\
99,71 ; \\
98,09\end{array}$ & $\begin{array}{l}\text { Wen, et al; } \\
2014\end{array}$ \\
\hline $\begin{array}{c}\mathrm{Ag}^{+1}, \mathrm{~Pb}^{+2}, \\
\mathrm{Hg}^{+2}, \\
\mathrm{Cu}^{+2}, \mathrm{Cr}^{+6}, \\
\mathrm{Fe}^{+3}, \mathrm{Fe}^{+2}\end{array}$ & $\begin{array}{l}10 \\
\text { excepto } \\
\text { Cr, que } \\
\text { es } 3\end{array}$ & $2 \mathrm{mg} / \mathrm{L}$ & $\begin{array}{c}20 \\
\min \end{array}$ & 30 & $\begin{array}{l}\text { Eliminación de iones } \\
\text { metálicos tóxicos de las } \\
\text { aguas residuales por } \\
\text { fotocatálisis de } \\
\text { semiconductores }\end{array}$ & $\begin{array}{c}14,1 ; 10,5 \\
13,3,7,1 ; \\
28,3 ; 95,2 ; \\
6,4\end{array}$ & $\begin{array}{l}\text { Dingwang, } \\
\text { et al; } 2013\end{array}$ \\
\hline $\mathrm{Cr}^{+6}$ & 2 & $150 \mathrm{mg} / \mathrm{L}$ & $\begin{array}{c}25 \\
\min \end{array}$ & 25 & $\begin{array}{l}\text { Fotocatálisis en el } \\
\text { nuevo sistema hetero- } \\
p-\mathrm{CuCO}_{2} \mathrm{O}_{4} / n- \\
\mathrm{TiO}_{2} \text { en presencia de } \\
\text { ácido tartárico. }\end{array}$ & $76-91$ & $\begin{array}{l}\text { Kebir, et } \\
\text { al; } 2015\end{array}$ \\
\hline
\end{tabular}

$\mathrm{t}_{\mathrm{Tr}}:$ Tiempo de tratamiento

en que se emplea la fotocatálisis para remover metales pesados.

\subsection{Técnicas No Convencionales.}

\subsubsection{Adsorbentes de Bajo Costo y Nuevos Adsorbentes}

La adsorción es un proceso de transferencia de masa por el cual una sustancia se transfiere desde la fase líquida a la superficie de un sólido. El pro- ceso de sorción describe en realidad un grupo de procesos, que incluyen la adsorción y las reacciones de precipitación. Recientemente, la adsorción se ha convertido en uno de las técnicas alternativas de tratamiento para las aguas residuales cargadas con metales pesados (Wan Ngah, et al; 2011). Se emplea un amplio espectro de materiales biológicos, especialmente las bacterias, algas, levaduras y hongos han recibido una atención creciente para la eliminación y recuperación de metales pesados, debido a su buen rendimiento, bajo 
coste y grandes cantidades disponibles (Wan \& Chen, 2009; Anastopoulos \& Kyzas, 2015).

2.2.2 Adsorción de metales pesados por materiales naturales agrícolas e industriales. Los procesos de adsorción están siendo ampliamente utilizados por varios investigadores para la eliminación de metales pesados. Los flujos de residuos y carbón activado se han usado con frecuencia como un adsorbente. A pesar de su amplio uso en las industrias de tratamiento de agua y aguas residuales, el carbón activado se mantiene como un material costoso. En los últimos años, la necesidad de métodos seguros y económicos para la eliminación de metales pesados de aguas contaminadas ha requerido interés de investigación hacia la producción de alternativas de bajo costo, disponible comercialmente. Por lo tanto, existe una urgente necesidad de que todas las fuentes posibles de los adsorbentes de bajo costo basados en la agroindustria deben explorarse y su viabilidad para la eliminación de metales pesados debe ser estudiada en detalle (Ahmed, 2013). A continuación en la tabla 16 se destacan 3 métodos viables para la adsorción o eliminación de metales pesados, utilizando residuo industrial, desechos agrícolas y minerales. Igualmente se destacan 2 métodos de remoción por fotocatálisis y absorción para eliminación simultánea.

2.2.3 Fitorremediación. Es el uso de las plantas y los microbios del suelo asociados para reducir las concentraciones o los efectos tóxicos de los contaminantes en los ambientes. Es una tecnología relativamente reciente y se percibe como rentable, eficiente, respetuoso del medio ambiente, y la tecnología solar-impulsado con buena aceptación del público (Ali et al,
2013). Estas fitotecnologías ofrecen numerosas ventajas en relación con los métodos fisicoquímicos que se usan en la actualidad, por ejemplo, su amplia aplicabilidad y bajo costo (Delgadillo et al, 2011). En esta revisión (Tabla 17) se presenta un panorama de las diversas técnicas fitocorrectivas empleadas para restaurar efluentes contaminados.

2.2.4 Biopolímeros. son industrialmente atractivos porque son capaces de reducir las concentraciones de iones metálicos de transición a concentraciones de partes por billón, son ampliamente disponibles y ambientalmente seguros (Barakat, 2011). Poseen un número amplio de diferentes grupos funcionales, tales como hidroxilos y aminas, que aumentan la eficiencia de la absorción de iones metálicos (Tabla 18).

2.2.5 Hidrogeles. Son polímeros hidrófilos reticulados capaces de ampliar sus volúmenes debido a su alta expansión en el agua. Por consiguiente, ellos son ampliamente utilizados en la purificación de las aguas residuales (Barakat, 2011; Hua, et al; 2014) Diversos hidrogeles se han sintetizado e igualmente investigado su comportamiento de adsorción de metales pesados, en la tabla 19 se presentan tres casos de estudio.

2.2.6 Ceniza Volante. Las cenizas volantes, generadas durante la combustión de carbón para la producción de energía, es un subproducto industrial que es reconocido como un contaminante ambiental, debido a su enriquecimiento en elementos traza potencialmente tóxica que se condensan del gas de combustión. Este material se ha reutilizado como un adsorbente de bajo costo para la eliminación de

Tabla 16. Adsorción o eliminación de metales pesados, utilizando residuo industrial, desechos agrícolas y minerales.

\begin{tabular}{|c|c|c|c|c|c|c|c|}
\hline \multirow[b]{2}{*}{ Especie } & \multicolumn{5}{|c|}{ Condiciones } & \multirow{2}{*}{$\begin{array}{c}\% \\
\text { Eficiencia } \\
\text { de remoción }\end{array}$} & \multirow[b]{2}{*}{ Ref. } \\
\hline & pH & $\begin{array}{l}\text { Concentra- } \\
\text { ción Inicial }\end{array}$ & $\mathbf{t T r}$ & $\begin{array}{c}\text { Temperatura } \\
{ }^{\circ} \mathrm{C} \\
\end{array}$ & pH & & \\
\hline $\begin{array}{l}\mathrm{Cr}^{3}, \mathrm{Cu}^{2} \\
\mathrm{~Pb}^{2+}, \mathrm{Zn}^{2} \\
+\end{array}$ & 5 & $\begin{array}{l}110 \mathrm{mg} / \mathrm{L} ; \\
110 \mathrm{mg} / \mathrm{L} ; \\
80 \mathrm{mg} / \mathrm{L} ; \\
80 \mathrm{mg} / \mathrm{L}\end{array}$ & $\begin{array}{c}45 \\
\min \end{array}$ & Ambiente & $\begin{array}{l}\text { Utilización de } \\
\text { residuos industriales } \\
\text { de celulosa y papel } \\
\text { "lodo de cal y cenizas } \\
\text { de caldera" }\end{array}$ & $\begin{array}{c}93 ; 99 ; 96 \\
99\end{array}$ & $\begin{array}{c}\text { Sthiannopkao } \\
\text { \& Sreesai, } \\
2009\end{array}$ \\
\hline $\begin{array}{l}\mathrm{Fe}, \mathrm{Pb}, \\
\mathrm{Cu}, \mathrm{Ni},\end{array}$ & 6 a 7 & $60 \mathrm{mg} / \mathrm{L}$ & $2 \mathrm{~h}$ & Ambiente & $\begin{array}{l}\text { Desechos agrícolas } \\
\text { como adsorbentes } \\
\text { "cascarilla de arroz" }\end{array}$ & $\begin{array}{l}99,25 ; \\
67,917 \\
98,177 \\
96,954\end{array}$ & Ahmed, 2013 \\
\hline $\begin{array}{l}\mathrm{Cr}^{3^{+}} \\
\mathrm{Zn}^{2^{+}} \\
\mathrm{Pb}^{2^{+}}\end{array}$ & $\begin{array}{l}4,05 \\
6,93 \\
3,86\end{array}$ & $\begin{array}{l}30 \mathrm{mg} / \mathrm{L} ; \\
30 \mathrm{mg} / \mathrm{L} ; \\
80 \mathrm{mg} / \mathrm{L} ; \\
10 \mathrm{mg} / \mathrm{L}\end{array}$ & $2 \mathrm{~h}$ & Ambiente & $\begin{array}{l}\text { Vermiculitas y el } \\
\text { efecto de la } \\
\text { precipitación }\end{array}$ & $\begin{array}{l}100 ; 99,7 ; \\
99,1 ; 98,38\end{array}$ & $\begin{array}{c}\text { Hikmet, et al; } \\
2014\end{array}$ \\
\hline
\end{tabular}

$\mathrm{t}_{\mathrm{Tr}}:$ Tiempo de tratamiento 
Tabla 17. Condiciones experimentales con diversos fitoplánctones.

\begin{tabular}{|c|c|c|c|c|c|c|c|}
\hline \multirow[b]{2}{*}{ Especie } & \multicolumn{5}{|c|}{ Condiciones } & \multirow{2}{*}{$\begin{array}{l}\text { \% Eficiencia } \\
\text { de remoción }\end{array}$} & \multirow[b]{2}{*}{ Ref. } \\
\hline & pH & $\begin{array}{c}\text { Concentración } \\
\text { inicial }\end{array}$ & $\mathrm{tTr}$ & $\begin{array}{c}\text { Temperatura } \\
{ }^{\circ} \mathrm{C}\end{array}$ & pH & & \\
\hline $\begin{array}{c}\mathrm{Pb}^{2+}, \\
\mathrm{Cd}^{2+}, \mathrm{Zn}^{2+}\end{array}$ & 4.84 & $\begin{array}{c}\text { no se midió } \\
\text { concentración } \\
\text { inicial }\end{array}$ & $3 \mathrm{~h}$ & Ambiente & $\begin{array}{c}\text { Biomasa de } \\
\text { Eichhornia crassipes }\end{array}$ & $\begin{array}{c}\text { Capacidad } \\
\text { de sorción } \\
26,32 \mathrm{mg} / \mathrm{g} \\
\mathrm{Pb} 12,60 \\
\mathrm{mg} / \mathrm{g} \mathrm{Cd} \\
12,55 \mathrm{mg} / \mathrm{g} \\
\mathrm{Zn}\end{array}$ & $\begin{array}{c}\text { Mahamadi \& } \\
\text { Nharingo, } 2010\end{array}$ \\
\hline $\mathrm{Cd}_{2+}^{2+}, \mathrm{Pb}$ & 5 & $5,0 \mathrm{~g} / \mathrm{L}$ & $12 \mathrm{~h}$ & Ambiente & $\begin{array}{c}\text { Jacinto de agua seca- } \\
\text { raíces }\end{array}$ & $\begin{array}{c}75 \% \text { para } \mathrm{Cd} \\
\text { y más de } 90 \% \\
\text { plomo }\end{array}$ & $\begin{array}{l}\text { Ibrahim, et al; } \\
2012\end{array}$ \\
\hline $\mathrm{Ni}, \mathrm{Zn}$ & - & $\begin{array}{l}10 \mathrm{~g}-3 \text { cada } \\
\text { uno }\end{array}$ & $12 \mathrm{~h}$ & Ambiente & $\begin{array}{l}\text { Corrientes de } \\
\text { productos de } \\
\text { pirolisis. Sorghum } \\
\text { bicolor (L.) }\end{array}$ & $\begin{array}{l}99 \% \mathrm{Ni} \mathrm{y} \\
98 \% \mathrm{Zn}\end{array}$ & $\begin{array}{l}\text { Chami, et al; } \\
2014\end{array}$ \\
\hline $\begin{array}{l}\mathrm{Cu}, \mathrm{Cd}, \\
\mathrm{Cr}, \mathrm{Ni}, \mathrm{Fe}, \\
\mathrm{Pb}, \mathrm{Zn}\end{array}$ & $9-11$ & 3 a $7,2 \mathrm{mg} / \mathrm{L}$ & $\begin{array}{c}14 \\
\text { días }\end{array}$ & Ambiente & $\begin{array}{l}P . \text { australis y } T \text {. latifol } \\
\text { ia en microcosmos. }\end{array}$ & $\begin{array}{c}78 \mathrm{Cu}, 60 \mathrm{Cd}, \\
68,1 \mathrm{Cr}, 73,8 \\
\mathrm{Ni} ; 80 \mathrm{Fe}, 61 \\
\mathrm{~Pb} \text { y } 61 \mathrm{Zn}\end{array}$ & $\begin{array}{c}\text { Kumari \& } \\
\text { Tripathi, 2015 }\end{array}$ \\
\hline $\mathrm{Cd}^{2+}$ & 8 & - & $\begin{array}{c}10 \\
\text { días }\end{array}$ & Ambiente & $\begin{array}{c}\text { Fitoplancton marino } \\
\text { Chaetoceros } \\
\text { calcitrans }\end{array}$ & $1055 \mathrm{mg} / \mathrm{g}$ & $\begin{array}{c}\text { Sjahrul \& } \\
\text { Arifin, } 2012\end{array}$ \\
\hline
\end{tabular}

$\mathrm{t}_{\mathrm{Tr}}:$ Tiempo de tratamiento

Tabla 18. Condiciones experimentales para remover iones de metales pesados empleando biopolímeros.

\begin{tabular}{|c|c|c|c|c|c|c|c|}
\hline \multirow[b]{2}{*}{ Especie } & \multicolumn{5}{|c|}{ Condiciones } & \multirow{2}{*}{$\begin{array}{c}\% \\
\text { Eficiencia } \\
\text { de remoción }\end{array}$} & \multirow[b]{2}{*}{ Ref. } \\
\hline & pH & $\begin{array}{c}\text { Concentración } \\
\text { Inicial }\end{array}$ & $\mathrm{tTr}$ & $\begin{array}{c}\text { Temperatura } \\
{ }^{\circ} \mathrm{C}\end{array}$ & pH & & \\
\hline $\begin{array}{l}\mathrm{Zn}^{2+} \\
\mathrm{Cu}^{2+} \\
\mathrm{Cd}^{2+} \\
\mathrm{Pb}^{2+}\end{array}$ & $\begin{array}{c}5,5 \mathrm{a} \\
6,5\end{array}$ & $\begin{array}{c}0,05 \mathrm{~g} \text { de } \\
\text { adsorbente y } 50 \\
\mathrm{ml} \text { de sln de } \\
\text { iones de metal } \\
\text { en } \\
\text { concentraciones } \\
\text { de } 100 \text { a } 1000 \\
\mathrm{mg} / \mathrm{L} .\end{array}$ & $\begin{array}{c}30 \\
\min \end{array}$ & $25-30$ & $\begin{array}{c}\text { Biopolímeros } \\
\text { celulósico injerto a } \\
\text { copolimeros }\end{array}$ & $\begin{array}{c}38,44, \\
46 \text { y } 48 \\
\text { respectiva- } \\
\text { mente }\end{array}$ & $\begin{array}{c}\text { Singha \& } \\
\text { Guleria, } 2014\end{array}$ \\
\hline $\begin{array}{l}\mathrm{Cr}^{3+} \\
\mathrm{Cr}^{6+}\end{array}$ & 2 & $\begin{array}{c}50 \text { ppm Cr y } \\
0,1 \mathrm{~g} \\
\text { absorbente } / 50 \mathrm{~m} \\
\text { L sln. }\end{array}$ & $6 \mathrm{~h}$ & Ambiente & $\begin{array}{c}\text { Lignina-quitosano } \\
\text { alcalino }\end{array}$ & $88-95$ & $\begin{array}{l}\text { Nair, et al, } \\
2014\end{array}$ \\
\hline $\begin{array}{l}\mathrm{Cd}^{2+} \\
\mathrm{Cu}^{2+} \\
\mathrm{As}^{5+}\end{array}$ & $\begin{array}{c}6,5 \mathrm{a} \\
7,5\end{array}$ & $50 \mathrm{ppm}$ & $\begin{array}{l}1 \mathrm{a} \\
2 \mathrm{~h}\end{array}$ & Ambiente & Quitosano Reticulado & $\begin{array}{l}\text { Capacidad } \\
\text { de absorción } \\
\text { de } 150,164 \\
\text { y } 230 \mathrm{mg} / \mathrm{g}\end{array}$ & Crini, 2005 \\
\hline $\begin{array}{l}\mathrm{Cd}^{2+} \\
\mathrm{Cu}^{2+} \\
\mathrm{Ni}^{2+}\end{array}$ & 5 a 6 & $\begin{array}{c}\text { 5g absorbente } \\
\text { en sln } 30 \mathrm{ppm} \\
\text { de metales }\end{array}$ & $10 \mathrm{~h}$ & Ambiente & $\begin{array}{c}\text { Aserrín injertado a } \\
\text { Poliacrilamida } \\
\text { carboxílada }\end{array}$ & 98,98 y 97 & $\begin{array}{l}\text { Gaey, et al; } \\
2010\end{array}$ \\
\hline
\end{tabular}

$\mathrm{t}_{\mathrm{Tr}}:$ Tiempo de tratamiento

compuestos orgánicos, gases de combustión y los metales pesados (Visa \& Chelaru, 2014); luego de aumentar su capacidad de adsorción mediante de la activación química y física (Ahmaruzzaman, 2010). En la tabla 20 se exponen las condiciones de tres estudios con estos materiales. 
Tabla 19. Empleo de hidrogeles para remover metales pesados y sus condiciones experimentales.

\begin{tabular}{|c|c|c|c|c|c|c|c|}
\hline \multirow[b]{2}{*}{ Especie } & \multicolumn{5}{|c|}{ Condiciones } & \multirow{2}{*}{$\begin{array}{c}\% \\
\text { Eficiencia } \\
\text { de remoción }\end{array}$} & \multirow[b]{2}{*}{ Ref. } \\
\hline & pH & $\begin{array}{c}\text { Concentración } \\
\text { Inicial }\end{array}$ & $\mathbf{t T r}$ & $\begin{array}{c}\text { Temperatura } \\
{ }^{\circ} \mathrm{C}\end{array}$ & pH & & \\
\hline $\begin{array}{l}\mathrm{Cu}^{2+} \\
\mathrm{Pb}^{2+} \\
\mathrm{Cd}^{2+} \\
\mathrm{Cr}^{3+}\end{array}$ & 4 a 6 & $100 \mathrm{ppm}$ & $24 \mathrm{~h}$ & 22 & $\begin{array}{l}\text { Hidrogel } \\
\text { funcionalizado con } \\
\text { sulfidrilo }\end{array}$ & $\begin{array}{c}\text { Capacidad } \\
\text { de absorción } \\
9.3,31.3 \\
17.7,5.2 \\
\mathrm{mg} / \mathrm{g} \\
\text { respectivame } \\
\text { nte. }\end{array}$ & $\begin{array}{l}\text { Hua \& Li, } \\
2014\end{array}$ \\
\hline $\begin{array}{l}\mathrm{Cu}^{2+}, \\
\mathrm{Cd}^{2+}, \\
\mathrm{Pb}^{2+} \\
\mathrm{Co}^{2+} \\
\mathrm{Ni}^{2+} \\
\mathrm{Cr}^{3+}\end{array}$ & 7 & $\begin{array}{c}0,2 \mathrm{~g} \mathrm{de} \\
\text { nanoabsorbente } \\
\text { en solución de } \\
\text { iones metálicos } \\
\text { a } 50 \mathrm{ppm}\end{array}$ & $2 \mathrm{~h}$ & Ambiente & $\begin{array}{l}\text { Incorporación de } \\
\text { thiacalix [4] areno } \\
\text { tetrasulfonato y } \\
\mathrm{Fe}_{3} \mathrm{O}_{4} \text { en } \\
\text { nanopartículas de } \\
\text { alginato de } \\
\text { sodio (Nanogel) }\end{array}$ & $\begin{array}{c}90.5,94.5 \\
99.8,75, \\
67.4 \mathrm{y} \\
79.2 \% \\
\text { respectivame } \\
\text { nte }\end{array}$ & $\begin{array}{c}\text { Mansour, et } \\
a l, 2014\end{array}$ \\
\hline $\begin{array}{c}\mathrm{Cu}^{2+}, \\
\mathrm{Zn}^{2+}, \\
\mathrm{Fe}^{3+}, \\
\mathrm{Cd}^{2+}, \mathrm{Cs}^{+}\end{array}$ & 5 a 6 & $\begin{array}{c}2-80 \mathrm{mM} \\
\mathrm{CuSO}_{4}\end{array}$ & $\begin{array}{c}3 \\
\text { días }\end{array}$ & Ambiente & $\begin{array}{l}\text { Hidrogel de celulosa } \\
\text { preparada utilizando } \\
\text { disolvente } \mathrm{LiOH} / \\
\text { urea }\end{array}$ & $\begin{array}{c}98 \%, 200, \\
100,110 \mathrm{y} \\
130 \mathrm{mg} / \mathrm{g} \\
\text { respectivame } \\
\text { nte }\end{array}$ & $\begin{array}{l}\text { Isobe, } \text { et al; } \\
2013\end{array}$ \\
\hline
\end{tabular}

$\mathrm{t}_{\mathrm{Tr}}:$ Tiempo de tratamiento

Tabla 20. Condiciones experimentales de absorción de metales pesados con cenizas volantes.

\begin{tabular}{|c|c|c|c|c|c|c|c|}
\hline \multirow[b]{2}{*}{ Especie } & \multicolumn{5}{|c|}{ Condiciones } & \multirow{2}{*}{$\begin{array}{c}\% \\
\text { Eficiencia } \\
\text { de remoción }\end{array}$} & \multirow[b]{2}{*}{ Ref. } \\
\hline & pH & $\begin{array}{c}\text { Concentración } \\
\text { Inicial }\end{array}$ & $\mathrm{tTr}$ & $\begin{array}{c}\text { Temperatura } \\
{ }^{\circ} \mathrm{C}\end{array}$ & pH & & \\
\hline $\mathrm{Hg}^{2+}$ & 5 & $\begin{array}{c}602 \mathrm{mg} \mathrm{Hg} / \mathrm{L} \mathrm{y} \\
\text { la concentración } \\
\text { de cenizas a } 100 \\
\mathrm{~g} / \mathrm{L}\end{array}$ & $\begin{array}{c}3 \\
\text { días }\end{array}$ & Ambiente & $\begin{array}{l}\text { Cenizas volantes } \\
\text { silico-aluminoso y } \\
\text { cenizas volantes- } \\
\text { sulfo cálcico. }\end{array}$ & $53-81$ & $\begin{array}{c}\text { Rio \& } \\
\text { Delebarre, } \\
2003\end{array}$ \\
\hline $\begin{array}{l}\mathrm{Pb}^{2+} \\
\mathrm{Hg}^{2+}\end{array}$ & 3 & $100 \mathrm{ppm}$ & $5 \mathrm{~h}$ & Ambiente & $\begin{array}{l}\text { Ceniza volante de } \\
\text { carbón }\end{array}$ & $34-17$ & $\begin{array}{c}\text { Prasetyo \& } \\
\text { Zakki, } 2013\end{array}$ \\
\hline $\mathrm{Cr}^{6+}$ & 2 & $50 \mathrm{ppm}$ & $1 \mathrm{~h}$ & 30 & $\begin{array}{l}\text { Cenizas volantes y } \\
\text { wollastonita }\end{array}$ & $\begin{array}{c}\text { Capacidad } \\
\text { de absorción } \\
2,92 \mathrm{mg} / \mathrm{g}\end{array}$ & $\begin{array}{c}\text { Ahmaruzzama } \\
\text { n, } 2010\end{array}$ \\
\hline
\end{tabular}

$\mathrm{t}_{\mathrm{Tr}}:$ Tiempo de tratamiento

\section{Conclusiones}

La regulación ambiental referente a vigilancia y control de los vertimientos industriales ha presentado un fortalecimiento evolutivo en los últimos años y ha evidenciado un notable incremento en los controles sobre los metales pesados; todo esto, debido al grado de peligrosidad de estos residuos y a su desbordado aumento y persistencia en las fuentes hídricas. El amplio espectro de tratamientos con diversos componentes y modificaciones que han sido estudiados para evaluar su eficiencia en la remoción de los metales pesados, también evidencia la preocupación de las autoridades y de la comunidad científica por pre- sentar soluciones a esta problemática; actualmente se ha desarrollado un elevado número de técnicas y de nuevos materiales con eficiencias prometedoras en procesos de absorción y adsorción, todas con el marcado objetivo de reducir costos energéticos y operacionales, que permitan su implementación y sostenibilidad.

\section{Referencias bibliográficas}

1. Ahmaruzzaman, M; 2010. A review on the utilization of fly ash. Progress in Energy and Combustion Science. 36, 327-363. 
2. Ahmed, H; 2013. Removal of heavy metals from wastewater using agricultural and industrial wastes as adsorbents. HBRC Journal. 9, 276-282.

3. Ali, H; Khan, E; Anwar, M; 2013. Phytoremediation of heavy metals - Concepts and applications, Chemosphere. 91, 869-881.

4. Alka, M; Kulkarni, S; Mungray, A; 2012. Removal of heavy metals from wastewater using micellar enhanced ultrafiltration technique: a review. Central European Journal of Chemistry, 10 (1), 27-46.

5. Al-Shannag, M;Al-Qodah, Z; Bani-Melhem, K; Rasool, M; Alkasrawi, M; 2015. Heavy metal ions removal from metal plating wastewater using electrocoagulation: Kinetic study and process performance, Chemical Engineering Journal. 260, 749-756.

6. Alijani, H; Beyki, M; Shariatinia, Z; Bayat, M; Shemirani, F, 2014. A new approach for one step synthesis of magnetic carbon nanotubes/diatomite earth composite by chemical vapor deposition method: Application for removal of lead ions. Chemical Engineering Journal. 253, 456-463.

7. Anastopoulos, I; Kyzas, G; 2015. Progress in batch biosorption of heavy metals onto algae, Journal of Molecular Liquids. 209, 77-86.

8. Baird, C; 2001. Química Ambiental. Editorial Reverté. University of Western Ontario. 622 pp.

9. Bakar, A; Halim, A; 2013. Treatment of automotive wastewater by coagulation-flocculation using polyaluminum chloride (PAC), ferric chloride $\left(\mathrm{FeCl}_{3}\right)$ and aluminum sulfate (alum). AIP Conference Proceedings, p. 14.

10.Barakat, M; 2011. New trends in removing heavy metals from industrial wastewater. Arabian Journal of Chemistry 4, 361-377.

11. Barczak, M; Michalak-Zwierz, K; Gdula, K; TyszczukRotko, K; Dobrowolski, R; Dabrowski, A; 2015. Ordered mesoporous carbons as effective sorbents for removal of heavy metal ions, Microporous and Mesoporous Materials. 211, 162-173.

12.Bi, Q; Zhang, Z; Zhao, C; Tao, Z; 2014. Study on the recovery of lithium from high $\mathrm{Mg} / \mathrm{Li}$ ratio brine by nanoltration Water Science \& Technology. 70 (10), 1690-1694.
13.Blue, L., Van Aelstyn, M.A., Matlock, M., Atwood, D; 2008. Low-level mercury removal from groundwater using a synthetic chelating ligand. Water Residual. 42, 2025-2028.

14.Bouhamed, F; Elouear, Z; Bouzid, J; Ouddane, B. 2015. Multi-component adsorption of copper, nickel and zinc from aqueous solutions onto activated carbon prepared from date stones. International Conference on Integrated Management of the Environment - ICIME 2014. Environmental Science Pollution Res, 6 pp.

15.Carrera, N; Domínguez, L; 2012. Remoción de metales pesados por métodos fisicoquímicos presentes en agua proveniente de una industria minera. Revista Sistemas Ambientales. 5(1), 27-40.

16.Cegłowski, M; Schroeder, G; 2015. Preparation of porous resin with Schiff base chelating groups for removal of heavy metal ions from aqueous solutions. Chemical Engineering Journal. 263, 402-411.

17.Cifuentes, L; García, P; Arriagada, J; Casas, J; 2009. The use of electrodialysis for metal separation and water recovery from $\mathrm{CuSO}_{4}-\mathrm{H}_{2} \mathrm{SO}_{4}$-Fe solutions. Separation and Purification Technology. 68 (1), 105-108.

18.Cifuentes, L; Ramírez, C; Crisostomo, G; Casas, J; 2011. Separación de especies de molibdeno por electrodiálisis Chemical Engineering Communications. 198, 805-814.

19. Chahar, A; Singh, A; 2014. Synthesis of novel tamarind 8-hydroxyquinoline-5-sulfonic acid (THQSA) resin and their application in industrial effluent treatment. International Journal of Pharmacy and Pharmaceutical Sciences. 6(8), 340-344.

20. Chami, Z; Amer, N; Smets, K; Yperman, J; Carleer, R; Dumontet, S; Vangronsveld, J; 2014. Evaluation of flash and slow pyrolysis applied on heavy metal contaminated Sorghum bicolor shoots resulting from phytoremediation, Biomass and Bioenergy. 63, 268-279.

21.Chen, Q., Luo, Z., Hills, C., Xue, G., Tyrer, M., 2009. Precipitation of heavy metals from wastewater using simulated flue gas: sequent additions of fly ash, lime and carbon dioxide. Water Residual. 43, 2605- 2614.

22.Chenar, S; Karbassi, A; Zaker, N; Ghazban, F; 2013. Electroflocculation of Metals during Estuarine Mixing 
(Caspian sea). Department of Environment, University of Tehran, p. 17.

23. Crini, G., 2005. Recent developments in polysaccharide-based materials used as adsorbents in wastewater treatment. Progress in Polymers Science. 30, 38-70.

24. Delgadillo, A; González, C; Prieto, F; Villagómez, J; Acevedo, O; 2011. Fitorremediación: una alternativa para eliminar la contaminación. Tropical and Subtropical Agroecosystems. 14 (2), 597-612.

25.Dingwang, C; Ajay, R; 2001. Removal of toxic metal ions from wastewater by semiconductor photocatalysis. Chemical Engineering Science. 56, 1561-1570.

26.Domenech, X; Peral, J; 2008. Química Ambiental de Sistemas Terrestres. Editorial Reverté. Barcelona, España. 239 pp.

27.Fan, L., Xu, X., Wang, G., Yang, J., Han, T; 2012. Experimental research for heavy metal lead and zinc smelting wastewater by electroflocculation. Advanced Materials Research. 534, 217-220.

28. Fu, F; Wang, Q; 2011. Removal of heavy metal ions from wastewaters: A review. Journal of Environmental Management. 92, 407-418.

29.Gaey, M., Marchetti, V., Clement, A., Loubinoux, B., Gerardin, P., 2010. Decontamination of synthetic solutions containing heavy metals using chemically modified sawdusts bearing polyacrylic acid chains. Journal of Wood Science. 46, 331-333.

30.Gherasim, C; Mikulášek, P; 2014. Influence of operating variables on the removal of heavy metal ions from aqueous solutions by nanofiltration Desalination. 343, 67-74.

31. González, M; Navarroa, R; Saucedoa, I; Avilaa, M; Prádanosb, P; Palaciob, L; Martínezb, F; Martínb, A. Hernández, A; 2006. Effect of phosphoric and hydrofluoric acid on the charge density of a nanofiltration membrane. Desalination. 200, 361-363.

32. Guastalli, A; Parrilla, R; Llorens, J; Mata, J; 2004. Application of Electrodialysis on Recovering Phosphoric Acid From an Industrial Rinsewater. En: Costa, J. 2004. Trends in Electrochemistry and Corrosion the Beginning of the 21st Century. Edicions Universitat Barcelona, 1241 pp.
33.Guo, L; Du, Y; Yi, Q; Li, D; Cao, L; Du, D; 2015. Efficient removal of arsenic from "dirty acid" wastewater by using a novel immersed multi-start distributor for sulphide feeding, Separation and Purification Technology. 142, 209-214.

34.Guerrero, J; Hernández, J; Pérez, R; 2006. Estudio preliminar del tratamiento del residual líquido (wl) de la empresa "comandante pedro sotto alba” moa nickel s.a. mediante el proceso de separación por membranas Tecnología Química. 26 (2), 83-93.

35.Hikmet, S; Turan, U; 2014. Removal of heavy metal ions from aqueous medium using kuluncak (Malatya) vermiculites and effect of precipitation on removal. Applied Clay Science. 95, 1-8.

36. Hua, R; Li, Z; 2014. Sulfhydryl functionalized hydrogel with magnetism: Synthesis, characterization, and adsorption behavior study for heavy metal removal. Chemical Engineering Journal. 249, 189-200.

37.Huang, J; Peng, L; Zeng, G; Li, X; Zhao, Y; Liu, L; Li, F; Chai, Q; 2014. Evaluation of micellar enhanced ultrafiltration for removing methylene blue and cadmium ion simultaneously with mixed surfactants, Separation and Purification Technology. 125, 83-89.

38.Huisman, J.L., Schouten, G., Schultz, C., 2006. Biologically produced sulphide for purification of process streams, effluent treatment and recovery of metals in the metal and mining industry. Hydrometallurgy 83, 106-113.

39. Ibrahim, H; Ammar, N; Soylak, M; Ibrahim, M; 2012. Removal of $\mathrm{Cd}(\mathrm{II})$ and $\mathrm{Pb}(\mathrm{II})$ from aquous solution using dry wáter hyacinth as a biosorbent. Spectrochimica Acta Part A: Molecular and Biomolecular Spectroscopy. 96 (1), 413-420.

40.Ioannou, L; Michael, C; Drosou, K; Xekoukoulotakis, N; Diamadopoulos, E; Fatta-Kassinos, D; 2013. Purificación de aguas residuales Bodega por ósmosis inversa y la oxidación del concentrado por energía solar foto-Fenton. Separation and Purification Technology. 118, 659-669.

41.Ismail, I; Fawzy, A; Abdel-Monem, N; Mahmoud, M; El-Halwany, M; 2012. Combined coagulation flocculation pre treatment unit for municipal wastewater, Journal of Advanced Research, 3, 331336. 
42.Isobe, N; Chen, X; Kim, U; Kimura, S; Wada, M; Saito, T; Isogai, A. 2013. TEMPO-oxidized cellulose hydrogel as a high-capacity and reusable heavy metal ion adsorbent, Journal of Hazardous Materials, 260, 195-201.

43.Jack, F; Bostock, J; Duarte, T; Harrison, B; Brosnan, $\mathrm{J}$; 2013. Electrocoagulation for the removal of copper from distillery waste streams. Institute of Brewing \& Distilling. Wiley Online Library, 342 pp.

44.Ji, Y; 2015. 16 - Membrane technologies for water treatment and reuse in the gas and petrochemical industries, En Woodhead Publishing Series in Energy, Advances in Membrane Technologies for Water Treatment Ed. Basile, A. \& Cassano, A. Woodhead Publishing, Oxford, 519-536.

45.Karnib, M; Kabbani, A; Holail, H; Olama, Z; 2014. Heavy Metals Removal Using Activated Carbon, Silica and Silica Activated Carbon Composite, Energy Procedia, Volume 50, 113-120.

46. Kebir, M; Trari, M; Maachi, R; Nasrallah, N; Bellal, B; Amrane, A; 2015. Relevance of a hybrid process coupling adsorption and visible light photocatalysis involving a new hetero-system $\mathrm{CuCo}_{2} \mathrm{O}_{4} / \mathrm{TiO}_{2}$ for the removal of hexavalent chromium, Journal of Environmental Chemical Engineering, 3, 548-559.

47.Khaled, B; Wided, B; Béchir, H; Elimame, E; Mouna, L; Zied, T; 2015. Investigation of electrocoagulation reactor design parameters effect on the removal of cadmium from synthetic and phosphate industrial wastewater, Arabian Journal of Chemistry, 1878-5352.

48.Khosa, M., Jamal, M., Hussain A., Muneer, M., Zia, K., Hafeez, S; 2013. Efficiency of Aluminum and Iron Electrodes for the Removal of Heavy Metals [(Ni (II), Pb (II), Cd (II)] by Electrocoagulation Method. Journal of the Korean Chemical Society. 57 (3), 15.

49.Kumar, P., Ramalingam, S., Sathyaselvabala, V., Kirupha, S., Murugesan, A., Sivanesan, S., 2012. Removal of Cd(II) from aqueous solution by agricultural waste cashew nut shell. Korean J. Chemical. Engineering. 29, 756-768.

50.Kumari, M; Tripathi, B; 2015. Efficiency of Phragmites australis and Typha latifolia for heavy metal removal from wastewater, Ecotoxicology and Environmental Safety. 112, 80-86.
51.Landaburu, J; Pongrácz, E; Keiski, R; 2011. Separation of cadmium and copper from phosphorous rich synthetic waters by micellar-enhanced ultrafiltration, Separation and Purification Technology. 81, 41-48.

52.Liu, S; Li, Z; Wang, C; Jiao, A; 2013. Enhancing both removal efficiency and permeate flux by potassium sodium tartrate (PST) in a nanofiltration process for the treatment of wastewater containing cadmium and zinc, Separation and Purification Technology.116, 131-136.

53.Liu, X; Lee, D; 2014. Thermodynamic parameters for adsorption equilibrium of heavy metals and dyes from wastewaters, Bioresource Technology. 160, 24-31.

54.Mahamadi, C; Nharingo, T., 2010. Utilization of Eichhornia Crassipes for the Removal of Pb(II), Cd(II), and Zn(II) from Aquatic Environments: An Adsorption Isotherm Study. Environmental Technology. 31(11), 1221-1228.

55. Mahmoud, A; Hoadley, A; 2012. An evaluation of a hybrid ion exchange electrodialysis process in the recovery of heavy metals from simulated dilute industrial wastewater. Water Research. 46, 3364-3376.

56. Mandel, k; Hutter, F; Gellermann, C; Sextl, G; 2013. Reusable superparamagnetic nanocomposite particles for magnetic separation of iron hydroxide precipitates to remove and recover heavy metal ions from aqueous solutions, Separation and Purification Technology. 109, 144-147.

57.Mansour, M; Mojerlou, F; Nazarzadeh, E; 2014. Nanogel and superparamagnetic nanocomposite based on sodium alginate for sorption of heavy metal ions. Carbohydrate Polymers. 106, 34-41.

58.MADS; 2015. Ministerio de Ambiente y Desarrollo Sostenible. República de Colombia. Resolución 0631 de 2015 "Por la cual se establecen los parámetros y los valores límites máximos permisibles en vertimientos puntuales a cuerpos de aguas superficiales y a sistemas de alcantarillado público, y se dictan otras disposiciones, $62 \mathrm{pp}$.

59.Méndez, R; Novelo, A; Coronado, V; Castillo, E; Sauri, M; 2008. Remoción de materia orgánica y metales pesados de lixiviados por flotación con aire disuelto. Ingeniería. 12, 13-19. 
60. Morante, G; 2002. Electrocoagulación de Aguas Residuales. Revista Colombiana de Física. 34 (2), 484-487.

61.Mubarak, N; Alicia, R; Abdullah, E; Sahu, J; Ayu Haslija, A; Tan, J; 2013. Statistical optimization and kinetic studies on removal of $\mathrm{Zn}^{2+}$ using functionalized carbon nanotubes and magnetic biochar, Journal of Environmental Chemical Engineering. 1, 486-495

62. Nair, V; Panigrahy, A; Vinua, R; 2014. Development of Novel Chitosan-Lignin Composites for Adsorption of Dyes and Metal Ions from Wastewater. Chemical Engineering Journal. 201, 1-51.

63. Nemerow, N; Dasgupta, A; 1998. Tratamiento de Vertidos Industriales Peligrosos. Ed. Dias de Santos. Madrid, 822 pp.

64.Nguyen, T; Ngo, H; Guo, W; Zhang, J; Liang, S; Yue, Q; Li, Q; Nguyen, T; 2013. Applicability of agricultural waste and by-products for adsorptive removal of heavy metals from wastewater. Bioresource Technology. 148, 574-585.

65.O’Connell, D; Birkinshaw, C; O’Dwyer, T; 2008. Heavy metal adsorbents prepared from the modification of cellulose: A review. Bioresource Technology. 99, 6709 - 6724.

66. Osei Boamah, P; Huang, Y; Hua, M; Zhang, Q; Wu, J; Onumah, J; Sam-Amoah, L; Osei Boamah, P; 2015. Sorption of heavy metal ions onto carboxylate chitosan derivatives-A mini-review, Ecotoxicology and Environmental Safety. 16, 113-120.

67.Paez, C; Taborda, G; 2006. La Fotocatálisis: Aspectos Fundamentales para una Buena Remoción de Contaminantes. Revista Universidad de Caldas, 7, 71-88.

68.Palakkeel, A; Veetil, D., Mercier, G., Blais, J. F., Chartier, M. et Tran, L; 2013. Simultaneous removal of $\mathrm{Cu}$ and PAHs from dredged sediments using flotation. J. Soils Sediments, 13 (8), 1502-1514.

69.Palanivelu, K; Vellaichamy, S; 2011. Preconcentration and separation of copper, nickel and zinc in aqueous samples by flame atomic absorption spectrometry after column solid-phase extraction onto MWCNTs impregnated with D2EHPA-TOPO mixture, Journal of Hazardous Materials, 185, 1131-1139.
70.Polat, H; Erdogan, D; 2007. Heavy metal removal from waste waters by ion flotation, Journal of Hazardous Materials. 148, 267-273.

71.Prado, M; Arruda, S; Ulson, G; Ulson, A; 2014. Study of lead (II) adsorption onto activated carbon originating from cow bone, Journal of Cleaner Production. 65, 342-349.

72. Prasetyo, E. Zakki, M; 2013. Removal of Hg and Pb in Aqueous Solution Using Coal Fly Ash Adsorbent. Procedia Earth and Planetary Science. 6, 377-382.

73.Ren, X; Chen, C; Nagatsu, M; Wang, X; 2011. Carbon nanotubes as adsorbents in environmental pollution management: A review, Chemical Engineering Journal. 170, 395-410.

74. Rio, S; Delebarre, A; 2003. Removal of mercury in aqueous solution by fluidized bed plant fly ash. Fuel 82. 153-159.

75. Sadyrbaeva, T; 2014. Recovery of Cobalt(II) by the Hybrid Liquid Membrane - Electrodialysis - Electrolysis Process, Electrochimica Acta. 133, 161-168.

76.SDAB; 2010. Concentraciones de Referencia para los Vertimientos Industriales Realizados a la Red de Alcantarillado y de los Vertimientos Industriales y Domésticos Efectuados a Cuerpos de Agua de la Ciudad de Bogotá. Primer Informe. Secretaria Distrital de Ambiente Bogotá (SDAB), Centro de Investigaciones en Ingeniería Ambiental - CIIA Departamento de Ingeniería Civil y Ambiental Universidad de los Andes, Bogotá. Colombia, p. 163.

77.Schwarze, M; Gross, M; Moritz, M; Buchner, G; Kapitzki, L; Chiappisi, L; Gradzielski, M; 2015. Micellar enhanced ultrafiltration (MEUF) of metal cations with oleylethoxycarboxylate, Journal of Membrane Science. 478, 140-147.

78.Singha, A; Guleria, A; 2014. Chemical modification of cellulosic biopolymer and its use in removal of heavy metal ions from wastewater. International Journal of Biological Macromolecules. 67, 409-417.

79.Sjahrul, M; Arifin, D; 2012. Phytoremediation of $\mathrm{Cd}^{2+}$ by Marine Phytoplanktons, Tetracelmis chuii and Chaetoceros calcitrans. Int. J. Chemestry. 4 (1), 69-74.

80. Spiro, T; Stigliani, W; 2006. Química Medioambiental. $2^{\circ}$ Edición. Editorial Pearson Prentice Hall. 504 pp. 
81.Sthiannopkao, S; Sreesai S; 2009 Utilization of pulp and paper industrial wastes to remove heavy metals from metal finishing wastewater. Journal of Environmental Management. 90, 3283-3289.

82.Taylor, J; Wiesner, M; 2002. Membranas. Capítulo 11. En: AWWA. Calidad y Tratamiento del Agua. Manual de suministros de Agua Comunitaria. McGraw Hill. Madrid, 707 - 779 pp.

83.Tu, K; Nghiem, L; Chivas, A; 2010. Boron removal by reverse osmosis membranes in seawater desalination applications, Separation and Purification Technology, 75, 87-101.

84. Visa, M; Chelaru, A; 2014. Hydrothermally modified fly ash for heavy metals and dyes removal in advanced wastewater treatment. Applied Surface Science 303, 14-22.

85.Wan Ngah, W; Teong, L; Hanafiah, M; 2011. Adsorption of dyes and heavy metal ions by chitosan composites: A review, Carbohydrate Polymers, 83, 1446 - 1456.

86. Wang, J; Chen, C; 2009. Biosorbents for heavy metals removal and their future, Biotechnology Advances. 27, 195 - 226
87.Wen, L; Jinren, N; Xiaochen, Y; 2014. Synergy of photocatalysis and adsorption for simultaneous removal of $\mathrm{Cr}(\mathrm{VI})$ and $\mathrm{Cr}(\mathrm{III})$ with $\mathrm{TiO} 2$ and titanate nanotubes. Water Research. 53, 12-25

88. Wang, X; Wang, Y; Wang, X; Liu, M; Xia, S; Yin, D; Zhao, J; 2011. Microwave-assisted preparation of bamboo charcoal-based iron-containing adsorbents for $\mathrm{Cr}(\mathrm{VI})$ removal. Chemical Engineering Journal. 174, 326 - 332.

89.Xu, Y; Zhang, J; Liang, Y; Zhou, J; Zhao, J; Ruan, X; Xu, Z; Qian, G; 2015. Synchronous cyanide purification with metals removal in the co-treatment of Zn-CN and Ni electroplating wastewaters via the $\mathrm{Ni}^{2+}$-assisted precipitation of LDH, Separation and Purification Technology. 145, 92-97.

90.Zewail, T; Yousef, N; 2015. Kinetic study of heavy metal ions removal by ion exchange in batch conical air spouted bed, Alexandria Engineering Journal,54, 83-90.

91.Zheng, Y; Xiong, C; Yao, C; Ye, F; Jiang, J; Zheng, X; Zheng, Q; 2015. Adsorption performance and mechanism for removal of Cd(II) from aqueous solutions by D001 cation-exchange resin. Water Science \& Technology, 69 (4), 833-839. 